듬 SCHOOL of GRADUATE STUDIES

EAST TENNESSEE STATE UNIVERSITY
East Tennessee State University Digital Commons@ East Tennessee State University

$8-2017$

\title{
Faces, Locations, and Tools: A Proposed Two- Stimulus P300 Brain Computer Interface
}

Marissa R. Jones

East Tennessee State University

Follow this and additional works at: https://dc.etsu.edu/etd

Part of the Cognitive Psychology Commons

\section{Recommended Citation}

Jones, Marissa R., "Faces, Locations, and Tools: A Proposed Two-Stimulus P300 Brain Computer Interface" (2017). Electronic Theses and Dissertations. Paper 3268. https://dc.etsu.edu/etd/3268

This Thesis - Open Access is brought to you for free and open access by the Student Works at Digital Commons @ East Tennessee State University. It has been accepted for inclusion in Electronic Theses and Dissertations by an authorized administrator of Digital Commons@ East Tennessee State

University. For more information, please contact digilib@etsu.edu. 
Faces, Locations, and Tools: A Proposed Two-Stimulus P300 Brain Computer Interface

\author{
A thesis \\ presented to \\ the faculty of the Department of Psychology \\ East Tennessee State University \\ In partial fulfillment \\ of the requirements for the degree \\ Master of Arts in Psychology \\ by \\ Marissa R. Jones \\ August 2017 \\ Eric W. Sellers, Ph.D., Chair \\ Matthew McBee, Ph.D. \\ Matthew Palmatier, Ph.D.
}

Keywords: EEG, Brain-Computer Interface 


\begin{abstract}
Faces, Locations, and Tools: A Proposed Two-Stimulus P300 Brain Computer Interface by

Marissa R. Jones

Brain Computer Interface (BCI) technology can be important for those unable to communicate due loss of muscle control. The P300 Speller allows communication at a rate up to eight selections per minute. Given this relatively slow rate of communication highly accurate classification is of great importance. Previous studies have shown that alternative stimuli (e.g., faces) can improve BCI speed and accuracy. The present study uses two new alternative stimuli, locations and graspable tools in a two-stimulus paradigm. Functional MRI studies have shown that images of familiar locations produce brain responses in the parahippocampal place area and graspable tools produce brain responses in premotor cortex. The current study shows that location and tool stimuli produce unique brain responses that can be used for classification in the two-stimulus paradigm. This study shows proof of concept for using two unique stimuli to improve speed and accuracy of the P300 Speller.
\end{abstract}




\section{TABLE OF CONTENTS}

Page

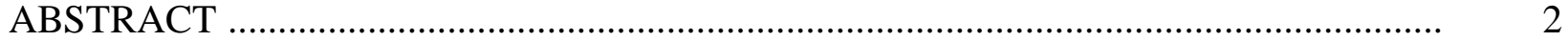

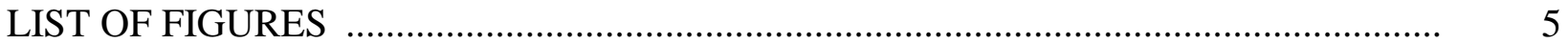

\section{Chapter}

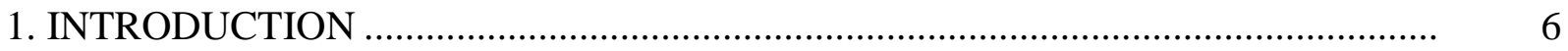

Brain Computer Interfaces ............................................................................... 6

Event-Related Potentials .....................................................................................

The P300 ERP Component ............................................................................... 8

The P300 Speller............................................................................................. 10

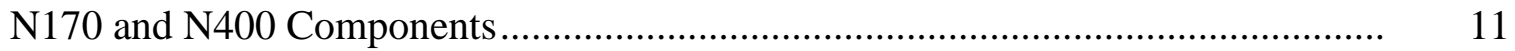

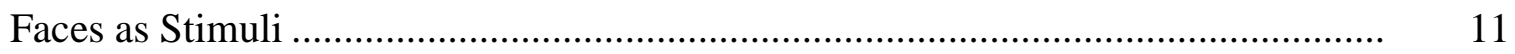

Facial Fusiform Area and Parahippocampal Place Area ......................................... 14

Project Overview ……………………........................................................ 15

2. EXPERIMENT 1 ...............................................................................................

Experiment 1 Methods ....................................................................................... 17

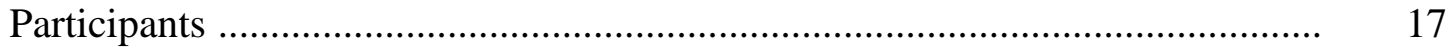

Data Acquisition and Processing ................................................................... 18

Experimental Stimuli ...................................................................................... 18

Experiment Procedure and Design................................................................ 19

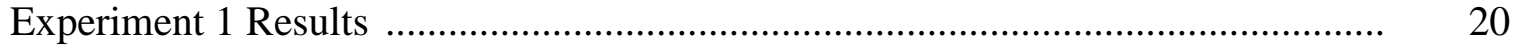

Statistical Analyses .................................................................................. 20

Waveform Analysis .................................................................................. 20

Experiment 1 Discussion .......................................................................... 23

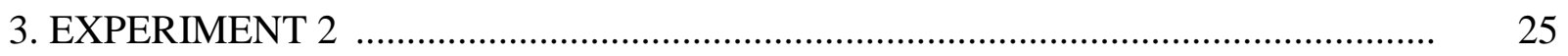

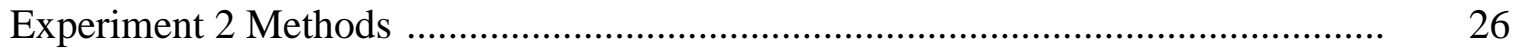

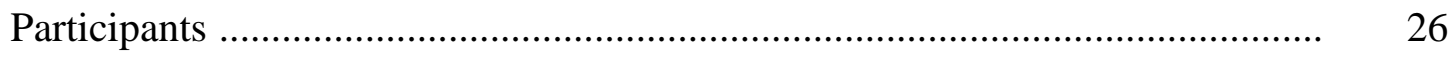

Data Acquisition and Processing ................................................................... 26 


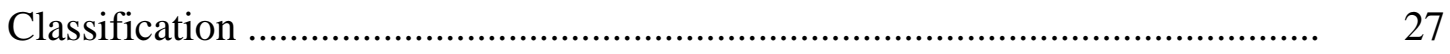

Experiment Procedure and Design................................................................ 28

Experiment 2 Results ........................................................................... 30

Statistical Analyses ................................................................................... $\quad 30$

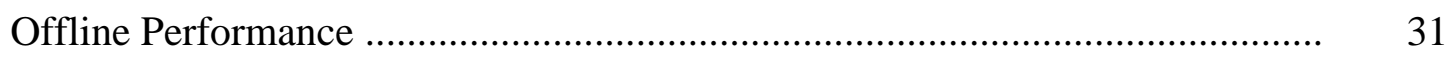

Waveform Analysis .................................................................................. 35

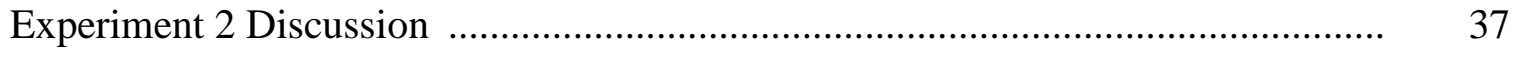

4. EXPERIMENT 3 _........................................................................................ 39

Graspable Objects as stimuli......................................................................... 39

Experiment 3 Methods ................................................................................. 41

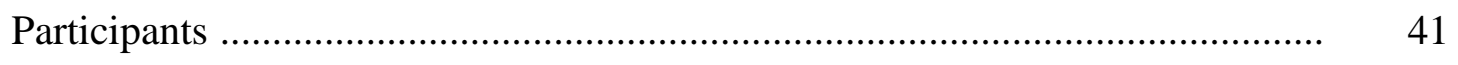

Data Acquisition and Processing ..................................................................... 41

Classification ......................................................................................... 41

Experiment Procedure and Design....................................................................... 41

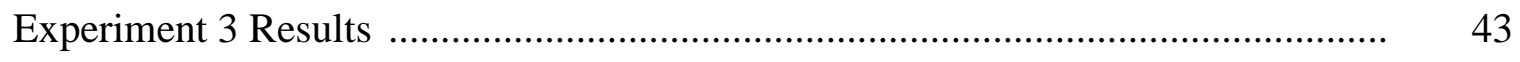

Statistical Analyses ......................................................................................

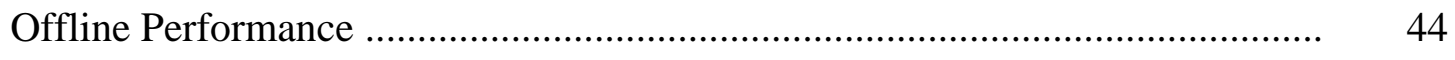

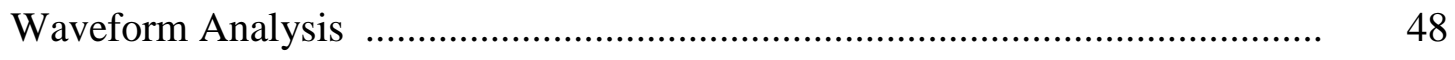

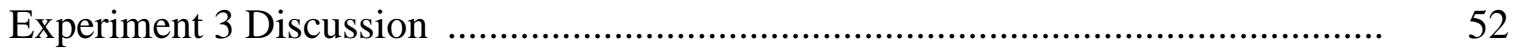

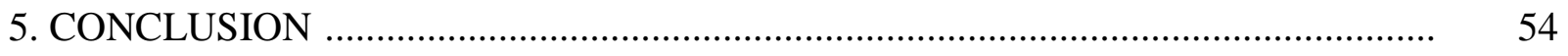

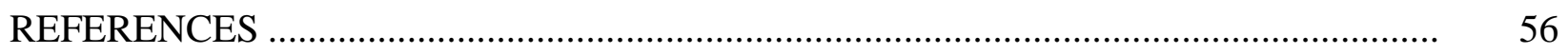

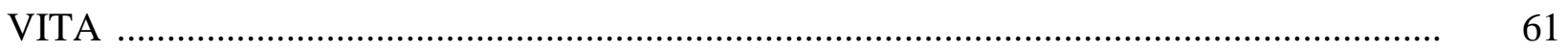




\section{LIST OF FIGURES}

Figure

Page

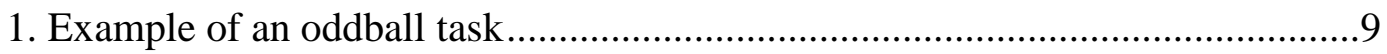

2. Example 6x6 checker-board paradigm ......................................................10

3. Example 6x6 matrix row-column paradigm presenting images of faces..........12

4. Example of two-stimulus paradigms .........................................................14

5. Examples of images used for facial stimuli and location stimuli ....................19

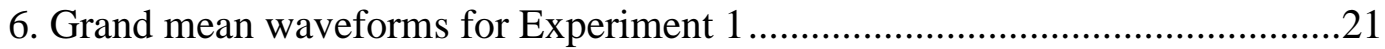

7. Examples of matrices of the stimulus presentation conditions ........................29

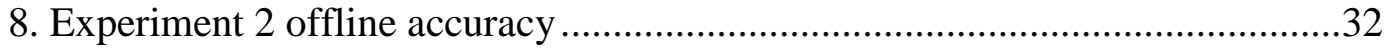

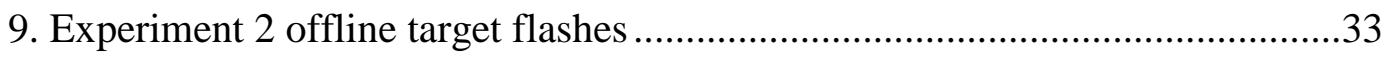

10. Experiment 2 offline selections per minute ...............................................

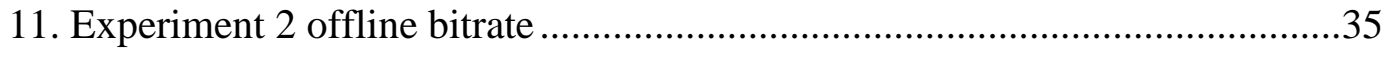

12. Grand mean waveforms for Experiment 2 ............................................36

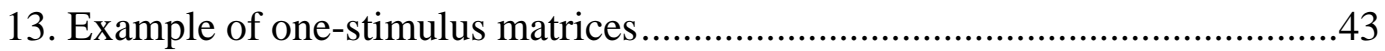

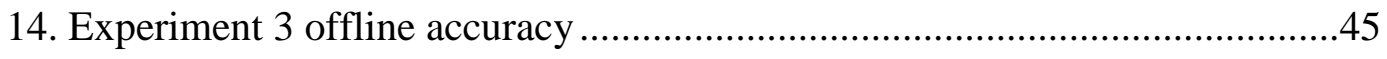

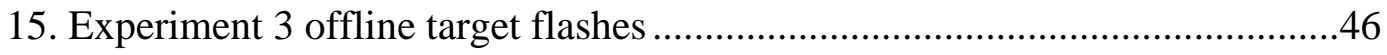

16. Experiment 3 offline selections per minute ...........................................4

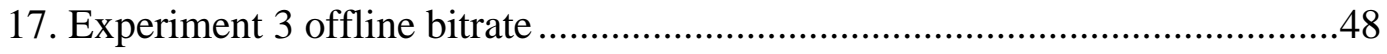

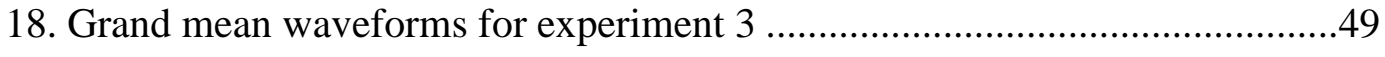




\section{CHAPTER 1}

\section{INTRODUCTION}

\section{$\underline{\text { Brain Computer Interfaces }}$}

Brain Computer Interfaces (BCIs) involve the measurement of neural signals produced by the electrical activity of the brain, a method or algorithm applied to decode these signals, and a systematic method for applying the decoded signals to a behavior (Sajda, Müller, \& Shenoy, 2008). Since researchers discovered the ability to apply these signals to operate an external device, the potential applications of BCI technology continues to grow. The uses of these recorded signals to operate BCIs can range from controlling external devices such as a robotic arm, to moving computer cursors, to creating works of art known as "Brain-Painting" (Münßinger et al., 2010; Velliste, Perel, Spalding, Whitford, \& Schwartz, 2008). These types of devices have the potential to be useful for individuals who have lost limbs or muscle movement that is necessary for daily functioning.

Presently, BCIs have been found to be particularly effective systems of communication for individuals who lost their ability to communicate due to loss of muscle control. Despite being unable to move or communicate, these individuals may retain cognitive functioning, and using BCIs can help bridge the gap between having thoughts and an inability to communicate those thoughts. Loss of movement and communication, as one can imagine, can take a toll on both these individuals and their loved ones. Those who develop amyotrophic lateral sclerosis (ALS), brainstem stroke, or severe traumatic brain injury who must now rely on a caretaker for day to day activities can benefit from using these systems of communication, as they require no muscle movement (Sellers, Krusienski, McFarland, Vaughan, \& Wolpaw, 2006). The P300 Speller, for 
examples, is a BCI communication method that can allow severely disabled individuals to convey their thoughts without having to rely on crucial muscle movement.

The present state of functional BCI research involves improving upon the systems that are used in laboratories, and to make these systems more accessible and functional for in-home use (Vaughn et al., 2006). Since the first developed systems, there has been great improvement on the operation of these BCIs, but there are still more improvements to be made to increase both functionality and usability. Increased speed and accuracy of word selection, information transfer rates, and being more user friendly are only some of the areas in which improvements can be made to benefit those who must rely on these systems to communicate. Over the past few years, researchers have taken many different approaches in the attempt to improve $\mathrm{BCI}$ functionality. Altering the presentation of stimuli, enhancing signal processing, improving signal acquisition and classification, and examining cognitive variables that may affect performance have been of particular interest for BCI researchers (Krusienski, Sellers, McFarland, Vaughn, \& Wolpaw, 2008). In addition, increasing signal-to-noise ratio of recorded ERPs and testing novel stimulus paradigms for ERP BCIs have become crucial aspects of BCI research (Kubler, Kotchoubey, Kaiser, Wolpaw, \& Birbaumer, 2001).

\section{$\underline{\text { Event-Related Potentials }}$}

The neural activity in non-invasive BCIs used for communication is recorded using electroencephalogram (EEG). Event related potentials (ERPs) are electrical responses elicited in reaction to an internal or external event, and different ERPs are elicited by attending to various types of stimuli, such as an auditory or visual stimulus (Vallabhaneni, Wang, \& He, 2005). ERPs are a series of positive and negative deflections that are identified by the time point at which the 
reaction to a stimulus is elicited. A vast amount of ERP research has been conducted over the years to examine cognitive processes that may provide insight into the relationship between human consciousness and behavior. Research into these cognitive processes via ERPs are observed using EEG by time-locking ERP responses that correspond to stimulus events (Fabiani, Gratton, Karis, \& Donchin, 1987). Several instances of the ERP response are recorded over multiple trials, or stimulus events. The recorded ERPs from multiple trials are averaged to produce an ERP because ERPs are small signals relative to the ongoing EEG.

ERPs are often categorized as either exogenous or endogenous in relation to how a stimulus is processed. Exogenous ERPs are typically involuntary responses to the physical properties of a stimulus and occur between 0 and $75 \mathrm{~ms}$ post stimulus presentation. Endogenous ERPs are more representative of the psychological and behavioral processes that are associated with a stimulus and typically occur approximately 100ms post stimulus presentation (Hillyard \& Kutas, 1983) By identifying the time-locked responses that appear to occur in response to specific stimuli, insight may be gained as to how and why certain cognitive processes occur.

\section{$\underline{\text { The P300 ERP Component }}$}

The P300 ERP was discovered in 1965 by Sutton, Braren, Zubin, and John, and is elicited by attending to rare occurring stimuli. The P300 is a positive component that occurs approximately 250 to $400 \mathrm{~ms}$ following the presentation of stimuli. Peak amplitudes for the P300 are best observed at parietal and central electrode locations (Picton, 1992). Donchin and Coles (1988) refer to the elicitation of a P300 component as context updating. Context updating is identified as the process in which an individual is presented with a stimulus, and then that individual goes through an evaluative process in which he or she assesses whether the presented 
stimulus is consistent with previous stimulus presentations. When a new or rare stimulus is presented, cognitive evaluative processes occur (context updating), and a P300 ERP is elicited in response to the change of stimuli.

One of the most often cited research paradigms examining the P300 component is what is known as the oddball task (Fabiani et al., 1987). In an oddball task, a participant is presented two different types of stimuli occurring at different frequencies, as shown in Figure 1.

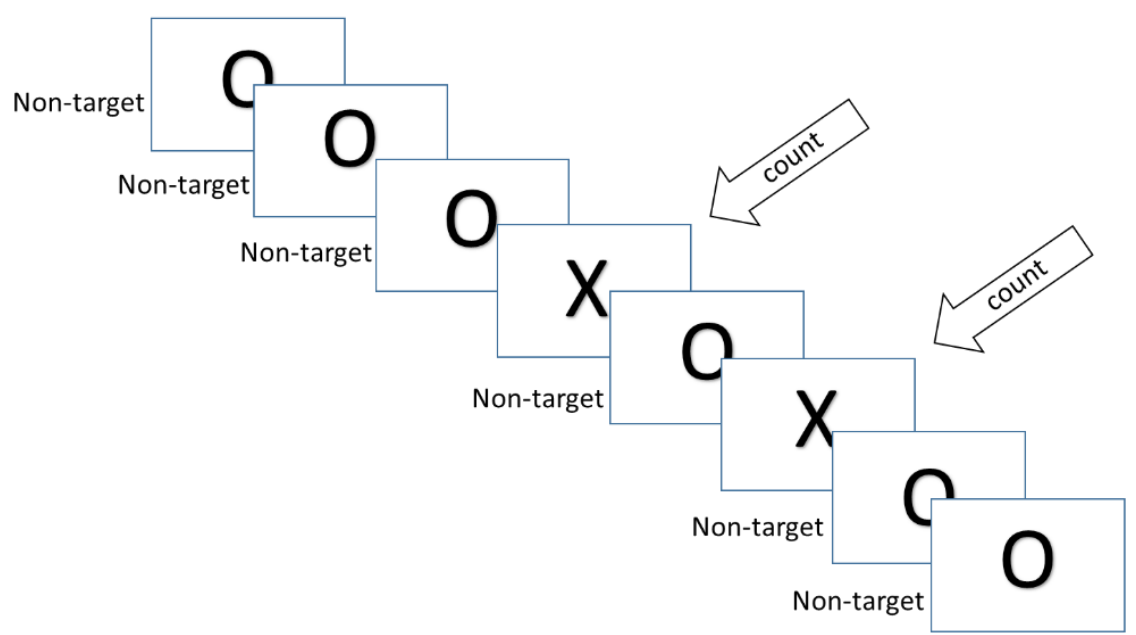

Figure 1. Example of an oddball task

For example, one stimulus type (e.g., O), is presented randomly $80 \%$ of the time. The other stimulus type, (e.g., X) is presented less frequently at $20 \%$ of the time. Participants are instructed to pay attention to X's and to attempt to ignore the frequently shown O's. Each time the participants see the infrequently shown X's, the P300 ERP is elicited in relation to attending to that rare occurring stimulus. 


\section{The P300 Speller}

The P300 speller is a method of BCI communicated that operates based on an oddball paradigm. The P300 speller displays a matrix of letters, numbers, and computer commands, like that of a computer keyboard (see Figure 2). Groups of items in the matrix are intensified or 'flash' at random intervals. In most standard P300 Speller BCIs, the 'flash' can consist of changing from grey to white, change from a different color to white, or will disappear and reappear. For the participant to make a character selection from the matrix, the participant pays close attention to the letter or character he or she would like to select. Each time the character of interest flashes, the participant updates a mental count of the character flash. When the participant attends to each individual flash of the desired character, a P300 response is elicited. The P300-Speller detects and examines these P300 responses, and is then able to discriminate between target characters versus non-target characters (i.e., letters the participant is trying to select versus letters the participant is not trying to select).

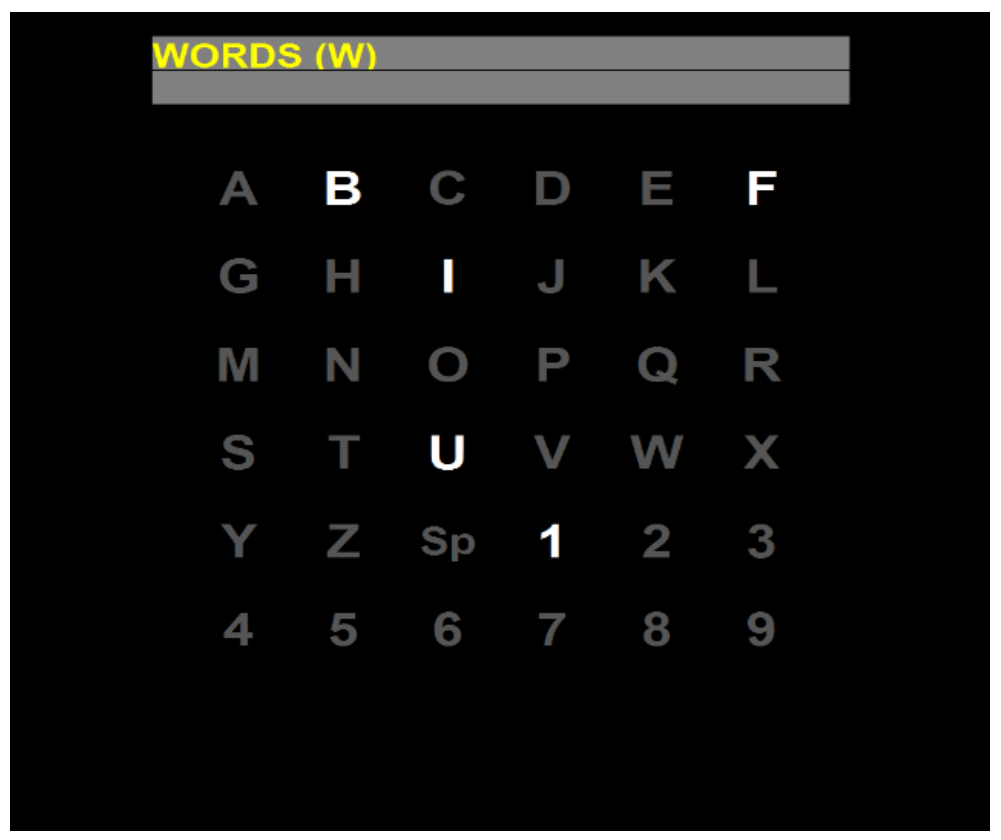

Figure 2. Example $6 x 6$ checker-board paradigm: showing a group of characters flashing. 


\section{$\underline{\text { N170 and N400 Components }}$}

Positive and negative ERPs are elicited, or are more robust, in response to specific stimulus types and events. Two negative components referred to as the N170 and N400 components have been shown to occur when participants recognize and process facial information. The N170 occurs in response to observing faces at lateral temporal electrode positions and occurs approximately 170ms following stimulus presentation (Bentin, 1996; Eimer, 2000). The N400 occurs approximately between 200ms and 600ms (Kutas \& Federmeier, 2011) over the right hemisphere electrode positions. Both of these components have been observed using unaltered facial images, inverted facial images, and even pictures of faces with drawn in facial features (Chen, Jin, Wang, \& Cichocki, 2015; Jin,, Daly, Zhang, Wang, \& Cichocki, 2014).

\section{Faces as Stimuli}

Over the years many studies have sought to improve the speed and accuracy of P300 BCI systems through means of stimulus presentation. The use of familiar faces as potential stimuli for P300-Speller character matrices was first implemented by Kaufmann, Schulz, Gruzinger, and Kubler (2011). Their hypothesis was that the components elicited by face stimuli would provide additional ERP information to augment the P300 ERP. Thus, the paradigm could increase signalto-noise ratio through the addition of the N170 and N400 ERP components. It was thought that the additional information would create a more robust and detectable response, resulting in improved overall BCI performance. Kaufmann et al., (2011) superimposed the familiar, famous image of Albert Einstein sticking his tongue out over characters within the BCI matrix. In each 
sequence of character flashes, the image itself would flash over the characters in the matrix, as opposed to the matrix characters themselves flashing (see Figure 3).

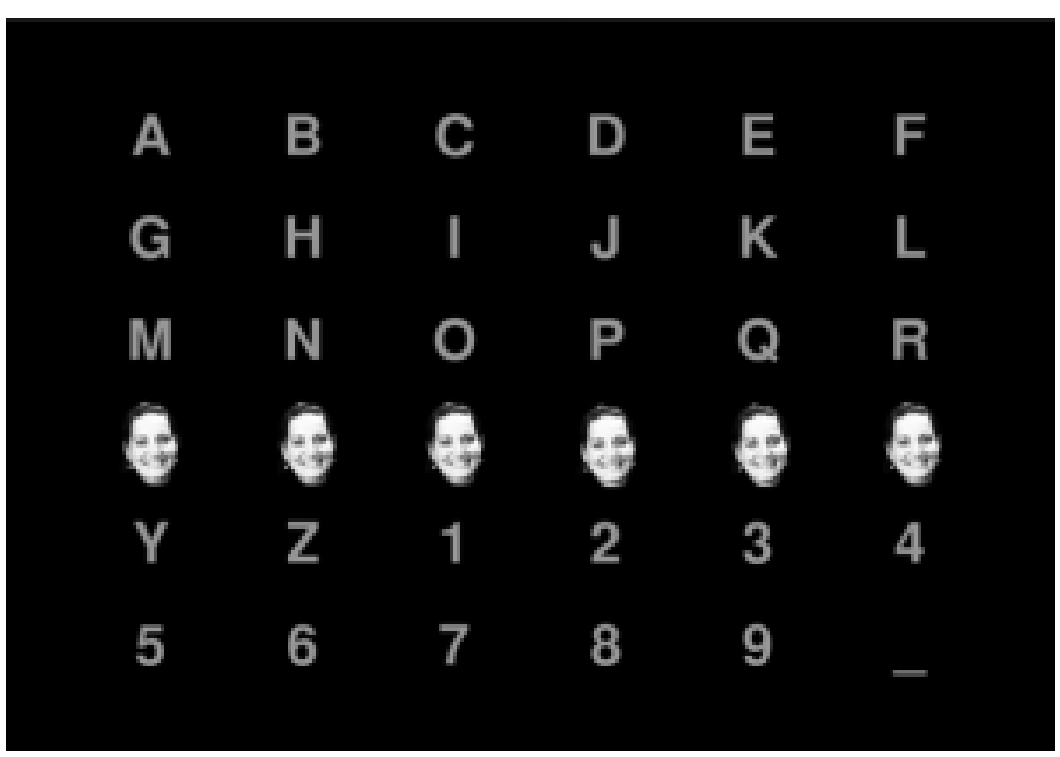

Figure 3. Example $6 x 6$ matrix row-column paradigm presenting images of faces (modified from Kaufmann et al. 2013).

The use of facial images to discriminate target flashes from non-target flashes, instead of traditional character flashes or symbol flashes, has been shown to increase both speed and accuracy of BCI use for patients with neurodegenerative disorders (Kaufmann et al., 2013). The rationale behind this increase in performance is thought to be due to face sensitive ERPs (Zhang, Zhao, Jin, Wang, \& Cichocki, 2012).

To create a control image, Kaufmann pixelated the Einstein image to retain facial characteristics while still creating an indistinguishable and obviously different image from the original Einstein face. This unique control condition was developed based on the findings of Takano, Komatsu, Hata, Nakajima, and Kansaku (2009), who found that physical features of stimuli such as chromatic differences, brightness, or contrast can impact BCI performance. Using a row-column flash pattern to investigate the influence of face stimuli of performance measures, 
they compared the face image paradigm to the pixelated image paradigm and the classic flashing of letter in the matrix typically used in the P300-Speller. The results of their findings indicated that flashing faces decreases the number of flashes required to reach high accuracy, in comparison to the classic character flashing that is typically used.

In 2013, Kaufmann et al. compared familiar personally-known faces to familiar famous faces based on the findings of Touryan, Gibson, Horne, and Weber (2011), who found that familiar family faces to elicit larger N400 responses than familiar famous faces. In the nondisabled sample, it was found that performance was significantly different between the classic character flash condition (CF) and the face conditions (FF) but there was no significant difference between the two FF conditions. In the patient sample, there were two patients who were unable to use the BCI using the CF stimuli. However, when using the FF stimuli, one patient could spell with an average of $80 \%$ accuracy for famous FF and $84 \%$ accuracy with personally known FF. The third patient was able to spell with $100 \%$ accuracy in both face conditions. These findings are important because it indicates that those who seem to be inefficient in using the BCI may be able to use the system when face images are used as stimuli.

To increase the speed of target selection, Kaufmann and Kubler (2014) introduced a paradigm that implemented a simultaneous presentation of two very different stimuli in the four quadrants of the matrix. The image of Einstein was presented in the top left and bottom right quadrants, and a yin-yang symbol was presented in the top right and bottom left quadrants. (see Figure 4). The two-stimulus presentation was compared to the standard row-column (see Figure 3 for comparison). The results showed that the two-stimulus paradigm was able to make selections more quickly than the one stimulus paradigm, despite a decrease in accuracy. 
Indicating that a two-stimulus paradigm could be a viable alternative to the more common single stimulus paradigm.

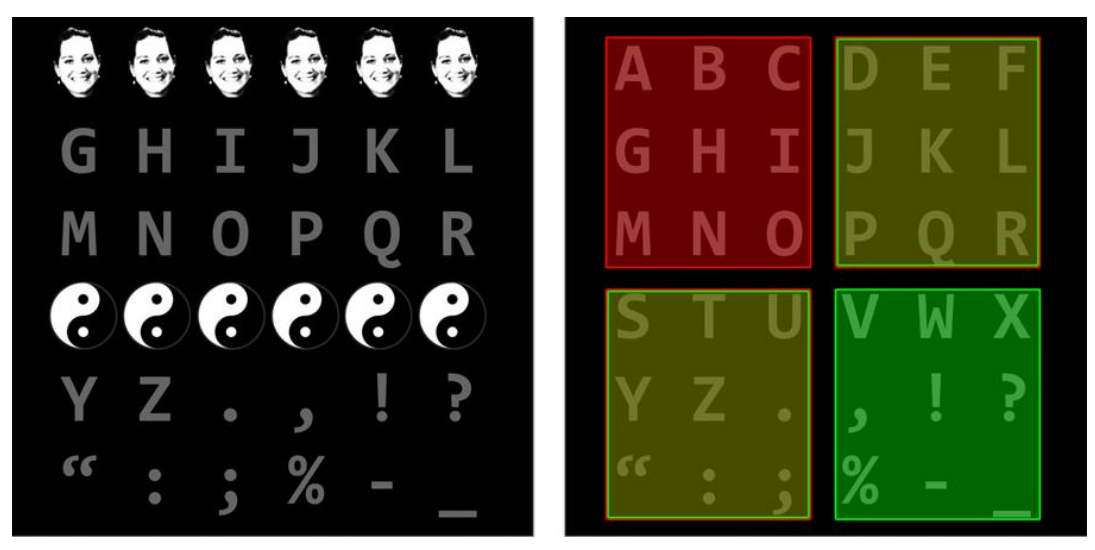

Figure 4. Example of two-stimulus paradigms (modified from Kaufmann et al. 2014).

\section{Facial Fusiform Area and Parahippocampal Place Area}

In addition to EEG research, several different neuroimaging techniques, such as positron emission tomography (PET) and functional magnetic resonance imaging (fMRI), have supported the idea that recognition and perception of different types of stimuli elicit different cognitive responses. There has been sufficient evidence that to indicate that the processing of facial stimuli and object stimuli, activate distinct brain regions. (Kanwisher, McDermott, \& Chun, 1997). The fusiform face area (FFA), which is comprised of the region in the mid-fusiform gyrus, is shown to be strongly activated by the viewing of faces compared to the viewing of objects (Haxby et al., 1991; Kanwisher et al., 1997; McCarthy, Puce, Gore, \& Allison, 1997; Sergent, Ohta, MacDonald, 1992; Tong, Nakayama, Vaughan, \& Kanwisher, 1998).

While neuroimaging studies indicate that the FFA responds selectively to facial stimuli, research has also demonstrated activation to images of buildings and scenes depicting locations in space in the parahippocampal gyrus referred to as the parahippocampal place area (PPA) (Agguire, Zarahn, \& D’Esposito, 1998; Epstein, Harris, Stanley, \& Kanwisher, 1999; Malach, 
Levy, \& Hasson, 2002). The PPA, located in the ventromedial surface of the temporal lobe, has been shown to respond selectively to houses and places, but not to objects or faces (Epstein \& Kanwisher, 1998). The strongest activation of the PPA was shown to occur in response to the viewing of complete images or photographs of scenes that depicted places, or even in images that showed empty landscapes with few discrete objects. Epstein et al. (1999) suggests that the spatial layout information of a scene may be itself be enough to activate the PPA, as the PPA may play a role in perceptual coding.

\section{$\underline{\text { Project Overview }}$}

The main purpose of the current project is to determine if a two-stimulus paradigm can increase BCI performance. The project consists of three experiments. Experiment 1 is a standard oddball study to examine the ERPs produced by facial and location stimuli. Experiment 2 tested facial and location stimuli in a two-stimulus BCI paradigm. Based on the findings of Experiment 2, Experiment 3 tested location and graspable object stimuli in a two-stimulus BCI paradigm.

The purpose of the oddball experiment (i.e., Experiment 1) was to determine if the facial and location stimuli would produce significantly different ERPs to be used in Experiment 2, the facial-location two-stimulus BCI paradigm. In the event that unique ERPs were produced by the two distinct stimuli, we hypothesized that the facial classifier would produce higher performance when applied to the facial stimuli, and the location classifier would produce higher performance to the location stimuli. In contrast, when each classifier is applied to the different class of stimuli performance would be reduced. To further enhance performance, Experiment 3 was conducted. Location and graspable object stimuli were used in a two-stimulus BCI paradigm. In this case it was hypothesized that the unique spatially distant locations activated by these two stimuli would 
result in more distinct ERPs, which would increase performance over the performance observed in the facial-location stimuli used in Experiment 2.

These studies, as discussed in the following sections, may provide a rationale for how and why a two-stimulus paradigm may be effective, and may also provide further evidence that the BCI system may detect ERPs that are specific to very different types of stimuli. Using an offline analysis, classifiers that are created specifically for facial stimuli and location stimuli are applied to collected online data. Predicted performance measures, such as accuracy, selections per minute, and bit rate are calculated to indicate whether each stimulus specific classifier would lead to increased performance when presenting corresponding stimuli on the BCI matrix. 


\section{CHAPTER 2}

\section{EXPERIMENT 1}

The premise of the current study relies on the BCI system's ability to detect differences in the ERPs produced by the two different stimulus types. Previous research has indicated that different types of stimuli can produce distinct cognitive responses. EEG research has provided substantial evidence showing that facial stimuli produce ERPs that are distinct from other types of visual stimuli, such as objects, symbols, houses and buildings, and locations in space (Haxby et al., 1991; Kanwisher et al., 1997; McCarthy et al., 1997; Sergent et al., 1992; Tong et al., 1998). Various neuroimaging studies have indicated that the viewing of images of familiar places, locations, and landscapes produce specific, localized activity that is not produced in response to viewing other types of stimuli (Agguire et al., 1998; Epstein et al., 1999; Malach et al., 2002). There has yet to be any research into the ERPs that may be produced specifically in response to viewing stimuli such as houses, buildings, and locations. To provide a rationale for the use of two different types of stimuli, an oddball study as described in Chapter 1, was conducted to investigate whether two different stimulus types would produce significantly different ERPs.

\section{Experiment 1 Methods}

\section{$\underline{\text { Participants }}$}

Twelve able-bodied participants (4 men, 8 women; age range 19-52) were recruited from East Tennessee State University. The study was approved by the East Tennessee State Institutional Review Board and each participant gave informed consent. 


\section{$\underline{\text { Data Acquisition and Processing }}$}

Electroencephalograph (EEG) was recorded using a cap (Electro-Cap International, Inc.) embedded with 32 tin electrodes. Channels were referenced to the right mastoid and grounded to the left mastoid. The signal was digitized at $256 \mathrm{~Hz}$ and bandpass-filtered to [0.5 Hz,30 Hz] by two 16-channel g.tec g.USBamp amplifiers. Data collection and stimulus presentation was performed by the BCI2000 open-source software suite (Schalk, McFarland, Hinterberger, Birbaumer, \& Wolpaw, 2004). Before the session, the impedance of each channel was reduced to below $40 \mathrm{k} \Omega$.

\section{$\underline{\text { Experimental Stimuli }}$}

Two types of images were used to examine the differences in ERPs associated with specific stimulus types (face specific and location specific). The facial stimuli that was used was the familiar, famous image of Albert Einstein sticking out his tongue in black and white (Figure 5a), as was used by previous BCI studies examining the effect of facial stimuli on BCI

performance (Kaufmann et al., 2011; Kaufmann et al., 2013; Kaufmann \& Kubler, 2014). The location stimulus chosen was an image of the White House (Figure 5b), also in black and white. The White House image was used because it not only included a famous landmark that is familiar to many people, but because it also included additional landscape information, such as trees and a fountain, that previous literature indicates would produce a cognitive response specific to processing location information. 

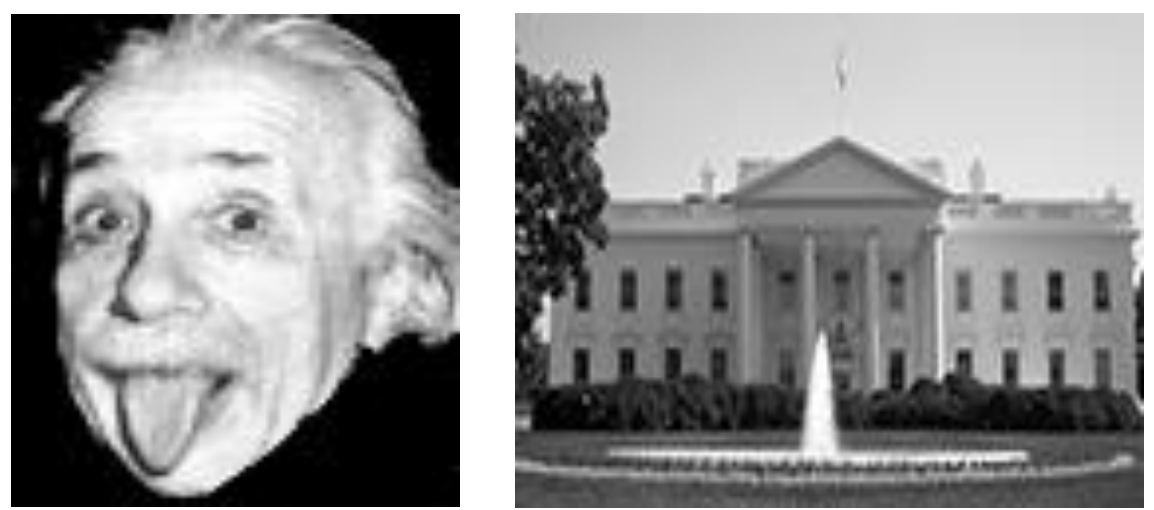

Figures $5 a$ (left) and $5 b$ (right). Examples of images used for facial stimuli and location stimuli.

In addition to examining the ERPs associated with image type, the effect of stimulus duration (the amount of time for one stimulus "flash") was also examined. The character flash duration in a standard BCI paradigm is typically $62.5 \mathrm{~ms}$, whereas paradigms using facial stimulus presentation use a flash duration of $187.5 \mathrm{~ms}$. The longer flash duration is used to allow for participants to have adequate time to recognize the images as faces. Therefore, four conditions were tested: Einstein image with flash duration of 62.5ms (Face 62.5), Einstein image with flash duration of $187.5 \mathrm{~ms}$ (Face 187.5), White House image with flash duration of 62.5 (White House 62.5), and White House image with flash duration of 187.5 (White House 187.5).

\section{Experiment Procedure and Design}

Participants completed one session testing the four different stimulus presentation conditions. Presentation order was counter-balanced to control for potential order effects. Each participant was seated in a chair approximately $90 \mathrm{~cm}$ away from a computer monitor. Participants were asked to complete an informed consent form and were debriefed on the experiment procedure. Following informed consent, each participant was fitted with a 32 electrode EEG cap. Participants were then instructed to focus their attention on a grey square presented in the center of the computer monitor. Participants were instructed to keep a mental 
count of the number of times the target stimulus (Face or White House) would flash in the center of the grey square. Three trial runs were completed for each of the four conditions for a total of 12 trials. Each trial consisted of $15(15 \%)$ target stimulus presentations and $85(85 \%)$ non-target stimulus presentations, for a total of 100 stimulus presentations in randomized order. Therefore, each condition had a total of 300 stimulus presentations.

\section{Experiment 1 Results}

\section{$\underline{\text { Statistical Analyses }}$}

Repeated measures analysis of the variance (ANOVA) was used to examine the four conditions. Comparisons between the four conditions were made based on the ERP amplitudes and latencies produced by the visual stimuli. ERP amplitudes and latencies produced by each of the four conditions were examined at two positive time windows and two negative time windows based on the calculated average waveform as shown in Figure 6.

\section{$\underline{\text { Waveform Analysis }}$}

The specified time windows examined for positive amplitudes and latencies were 150320ms and 350-550ms. The specified time windows to examine N170 and N400 amplitudes and latencies were sets to 128-195 for the N170 component, and 191-300 for the N400 component, determined by visual inspection of grand mean waveforms. 

$\mathrm{Cz}$
$\mathbf{P z}$
PO7
PO8
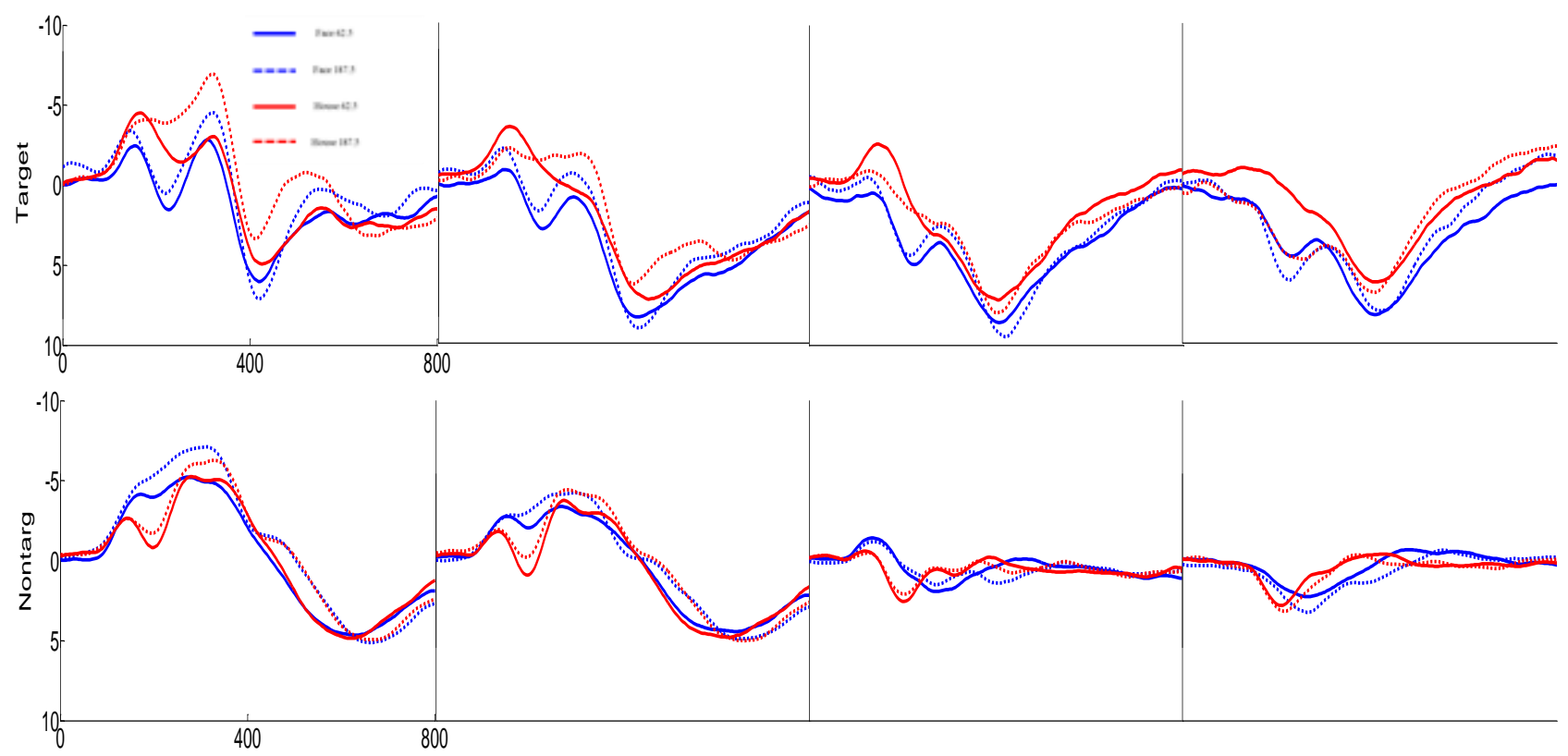

Figure 6. Grand mean waveforms for Experiment 1: Face 62.5 (blue solid), Face 187.5 (blue dashed), White House 62.5 (red solid), White House 187.5 (red dashed).

Repeated measures ANOVA examining positive amplitudes for time window 150-350ms at electrode location Pz found Mauchly's test of Sphericity to not be significant ( $\mathrm{p}=.152)$ indicating no violation of the assumption of sphericity. With sphericity assumed, the ANOVA indicated significant differences between the four conditions $F(3,33)=9.902, \mathrm{p}<.0001$. Post-hoc tests using Bonferroni corrections found significant differences for the comparison of positive amplitudes between Face 62.5 ( $\mathrm{M}=5.16, \mathrm{SD}=4.87)$ and White House $62.5(\mathrm{M}=2.05, \mathrm{SD}=3.7)$, $(\mathrm{p}=.039)$, comparison between Face 62.5 and White House 187.5 $(\mathrm{M}=1.75, \mathrm{SD}=3.57)(\mathrm{p}=.044)$, comparison between Face $187.5(\mathrm{M}=5,4.88)$ and White House $62.5(\mathrm{p}=.009)$, and comparison between Face 187.5 and White House 187.5 (p=.028). No differences, however, were found between the two Face conditions or between the two different White House conditions. 
Repeated measures ANOVA examining positive latencies for time window 150-320ms at electrode location Pz found Mauchly's test of Sphericity to not be significant ( $\mathrm{p}=.448$ ) indicating no violation of the assumption of sphericity. With sphericity assumed, the ANOVA indicated no significant differences between the latencies produced by Face $62.5(\mathrm{M}=263.7, \mathrm{SD}=47.4)$, Face 187.5 $(\mathrm{M}=238.6, \mathrm{SD}=44.4)$, White House 62.5 $(\mathrm{M}=267.6, \mathrm{SD}=59.8)$, and White House 187.5 $(\mathrm{M}=239.9, \mathrm{SD}=69), F(3,33)=1.474, \mathrm{p}=.239$.

Repeated measures ANOVA examining positive amplitudes for time window 350-550ms at electrode location Pz found Mauchly's test of Sphericity to be significant $(\mathrm{p}<.001)$ indicating a violation of the assumption of sphericity. Greenhouse-Geisser was used to correct for the assumption violation, and indicated no significant differences between the amplitudes of Face $62.5(\mathrm{M}=9.2, \mathrm{SD}=4.6)$, Face $187.5(\mathrm{M}=9.8, \mathrm{SD}=3.9)$, White House $62.5(\mathrm{M}=6.37, \mathrm{SD}=4)$, and White House 187.5 $(\mathrm{M}=6.5, \mathrm{SD}=5), F(1.271,13.977)=3.642, \mathrm{p}=.069$.

Repeated measures ANOVA examining positive latencies for time window 350-550ms at electrode location Pz found Mauchly's test of Sphericity to not be significant ( $p=.934)$ With sphericity assumed, the ANOVA indicated no significant differences between the latencies produced by Face $62.5(M=461.6, S D=41.6)$, Face $187.5(M=460.6, S D=39.9)$, White House $62.5(\mathrm{M}=482.4, \mathrm{SD}=52.4)$, and White House $187.5(\mathrm{M}=468, \mathrm{SD}=56.7), F(3,33)=.911, \mathrm{p}=.446$.

Repeated measures ANOVA examining negative amplitudes for time window 128-195ms at electrode location Pz found Mauchly's test of Sphericity to not be significant ( $\mathrm{p}=.278)$. With sphericity assumed, the ANOVA indicated no significant differences between the amplitudes produced by Face $62.5(\mathrm{M}=-5.1, \mathrm{SD}=3.4)$, Face $187.5(\mathrm{M}=-5.8, \mathrm{SD}=3.1)$, White House 62.5 $(\mathrm{M}=-7.4, \mathrm{SD}=3.2)$, and White House 187.5 $(\mathrm{M}=-6.1, \mathrm{SD}=3.2), F(3,33)=2.296, \mathrm{p}=.096$. 
Repeated measures ANOVA examining negative latencies for time window 128-195ms at electrode location Pz found Mauchly's test of Sphericity to not be significant $(p=.077)$. With sphericity assumed, the ANOVA indicated no significant differences between the latencies produced by Face $62.5(\mathrm{M}=160.5, \mathrm{SD}=20.6)$, Face $187.5(\mathrm{M}=155.6, \mathrm{SD}=22.5)$, White House $62.5(\mathrm{M}=165, \mathrm{SD}=20.3)$, and White House $187.5(\mathrm{M}=156.9, \mathrm{SD}=23.4), F(3,33)=1.178$, $\mathrm{p}=.333$.

Repeated measures ANOVA examining negative amplitudes for time window 191-300ms at electrode location Pz found Mauchly's test of Sphericity to not be significant $(\mathrm{p}=.170)$. With sphericity assumed, the ANOVA indicated no significant differences between the amplitudes

produced by Face $62.5(\mathrm{M}=-4, \mathrm{SD}=4.1)$, Face $187.5(\mathrm{M}=-4.5, \mathrm{SD}=3.5)$, White House $62.5(\mathrm{M}=-$ 5.2, $\mathrm{SD}=2.9)$, and White House $187.5(\mathrm{M}=-4.8, \mathrm{SD}=2.9), F(1.271,13.977)=3.462, F(3,33)$ $=.571, \mathrm{p}=.638$. Repeated measures ANOVA examining negative latencies for time window 191300ms at electrode location Pz found Mauchly's test of Sphericity to be significant, $(\mathrm{p}=.39)$, indicating a violation of the assumption of sphericity. Greenhouse-Geisser was used to correct for the assumption violation, and indicated no significant differences in the amplitudes of Face $62.5(\mathrm{M}=250, \mathrm{SD}=44.9)$, Face 187.5 $(\mathrm{M}=251.6, \mathrm{SD}=46)$, White House $62.5(\mathrm{M}=238.9$, $\mathrm{SD}=45.3)$, and White House $187.5(\mathrm{M}=254.2, \mathrm{SD}=35.4), F(1.792,19.708)=.406, \mathrm{p}=.650$.

\section{Experiment 1 Discussion}

Oddball data was collected to examine whether face stimuli and location stimuli would produce significantly different cognitive responses in the form of ERPs. This was to provide a rationale for using these specific stimulus types, the Einstein face and the White House location images, for Experiment 2. 
Results of Experiment 1 showed significantly higher amplitudes produced by two Face conditions than the two White House conditions at time window of $150-320 \mathrm{~ms}$, indicating a stronger P300 response to the Face image. While statistical analyses indicated a significant difference in the $\mathrm{P} 300$ responses produced by the two different images, there was no indication of a statistical difference between the Face image and the White House images for the N170 and N400 responses. This contrasts with previous research that has suggested that the N170 response, specifically, ought to be uniquely associated with processing of facial stimuli. The results of the statistical analyses, however, indicate that an N170 response occurred in response to viewing both the Face image and the White House image.

Despite there being an indication of only significant differences between the two image types in regards to the $\mathrm{P} 300$ response, it is possible that the BCI system may be sensitive enough to detect slight differences between the N170 and N400 responses produced by the two different image types. 


\section{CHAPTER 3}

\section{EXPERIMENT 2}

The results of the Experiment 1 showed that unique ERPs were produced by the facial and location stimuli. Thus, the BCI system may be capable of producing significantly different classification coefficients to discriminate between the two types of stimuli.

To be able to operate the P300 BCI, robust ERPs must be produced for the system to detect and translate the ERPs into character selections (Lotte, Congedo, L'ecuyer, Lamarche, \& Arnaldi, 2007). These ERPs, traditionally the P300 response, are used to discriminate target versus non-target characters. Kaufmann's research has provided support for the idea that the use of stimuli that elicit distinguishable components in addition to the P300 component can lead to increased rates of BCI performance. Studies in our own lab found no effect of stimulus type on performance; however, we did find significantly different ERPs produced by stimuli that are more complex than letters or numbers found in the BCI matrix.

The current study aims to provide proof of concept. Two stimuli that produce distinct ERPs could be used to improve upon current BCI classification methods. If a matrix is presented with two images conveying different types of information, such as an image of a familiar face or an image of a familiar location, a specific classifier could be created for each stimulus. The unique classifiers could then be used to identify target characters, based on the notion that these images produce different enough ERPs for the BCI to detect.

Previous studies indicate that the BCI can detect face-specific ERPs to improve classification methods. By superimposing an image of a face on half of the characters in the matrix, and superimposing another type of image on the other half of the characters in the matrix, 
two different classifiers could be made specifically for each image type. One classifier could detect the face-specific ERPs, while the other classifier could detect ERPs specific to the other stimulus type. This could allow the BCI system discriminate targets from non-targets faster than current methods by eliminating half of the characters in the matrix as potential targets. For example, if a participant wishes to select the letter A from the matrix, a familiar facial image is superimposed over the letters A, C, E, while an image of a familiar place or location is superimposed over the letters B, D, and F. The face classifier would detect the ERP responses of the participant in response to attending to the facial image, while the other classifier would detect ERPs that are specific to an image depicting a specific location. When the participant attends to the letter A, a P300, an N170, and an N400 are elicited. Since the N170 and N400 components are face specific, the system identifies the target as one of the "face" characters, A, C, or E, thereby eliminating the "location" characters B, D, and F.

\section{Experiment 2 Methods}

\section{$\underline{\text { Participants }}$}

Ten able-bodied participants (4 men, 6 women; age range 19-31) were recruited from East Tennessee State University. Four of the participants had prior BCI experience, the remainder of participants were naïve to BCI use. The study was approved by the East Tennessee State Institutional Review Board and each participant gave informed consent.

\section{Data Acquisition and Processing}

Electroencephalograph (EEG) was recorded using a cap (Electro-Cap International, Inc.) embedded with 32 tin electrodes. Channels were referenced to the right mastoid and grounded to the left mastoid. Only electrodes Fz, Cz, P3, Pz, P4, PO7, PO8, and Oz were used for the 
operation of the BCI (Krusienskiet et al., 2008). The EEG was digitized at $256 \mathrm{~Hz}$ and bandpassfiltered to [0.5 Hz, $30 \mathrm{~Hz}]$ by two 16-channel g.tec g.USBamp amplifiers. Data collection and stimulus presentation was performed by the BCI2000 open-source software suite (Schalk, McFarland, Hinterberger, Birbaumer, \& Wolpaw, 2004). Before the session, the impedance of each channel was reduced to below $40 \mathrm{k} \Omega$. Participants were seated approximately $90 \mathrm{~cm}$ away from a computer monitor that displayed an $8 \times 9$ matrix of letters and numbers. The speller matrix was adapted from a BCI2000 system used in patients' homes.

\section{$\underline{\text { Classification }}$}

The classification technique known as Stepwise Linear Discriminate Analysis (SWLDA) as described by Draper and Smith (1981) is a commonly used method to determine classification coefficients. SWLDA has previously been shown to be an efficient method of classification for BCI research (Farwell \& Donchin, 1988; Colwell Ryan, Throckmorton, Sellers, \& Collins, 2014; Krusienski et al, 2006; Krusienski et al, 2008; Sellers \& Donchin, 2006). By using forward regression and backward regression, the SWLDA algorithm selects the features that are extracted from the raw EEG signals that account for the most unique variance. Features are weighted by ordinary least-squares regression. Beginning with no features in the model, the feature that is the most statistically significant $(\mathrm{p}<0.1)$ is added to the model. A backward stepwise analysis then removes features that are found to be the least significant $(\mathrm{p}>0.15)$. There is a predetermined number of features included in the function, so that the process is repeated until reaching a maximum of 60 , or until there are no more features that meet the criterion for entry or removal. The SWLDA procedure provides classification coefficients, which are then applied to the average ERP responses during the online testing phase. 
To improve upon classification performance, multiple electrodes at various locations distributed over the scalp are used. A limitation to using a system with a larger number of electrodes is that the systems are more expensive and require more time to set up for at home users (Colwell et al., 2014). A filter method known as jumpwise selection is used to improve upon classification through optimal channel selection. Jumpwise selection uses a variant of SWLDA that selects electrodes instead of electrode specific features. Once the most statistically significant channels are selected, a SWLDA, as described above, is conducted on the specific features contained within the jumpwise defined electrode set. The advantage of jumpwise selection is that it reduces to the feature space to a unique set of features that are chosen from electrode locations that are optimized for each individual participant.

\section{Experiment Procedure and Design}

The image of Einstein sticking his tongue out and the image of the White House that were used in Experiment 1 as the facial and location stimuli were also used for Experiment 2. Each participant completed one session using three different presentations of stimuli, facial stimuli only (Face; Figure 7a), location stimuli only (White House; Figure 7b), and a matrix comprised of both Faces and White Houses displayed using a checkerboard flash pattern (Figure $7 c)$. 

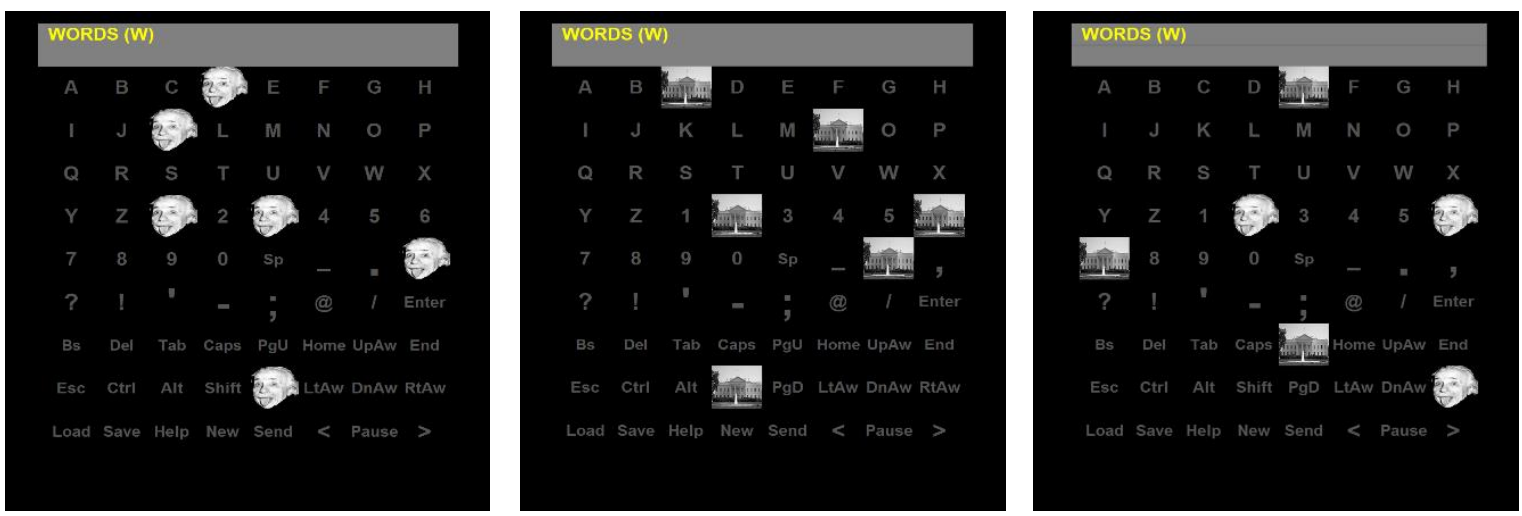

Figure $7 a$ (left), $7 b$ (middle), and $7 c$ (right). Examples of matrices of the stimulus presentation conditions.

Each participant completed on experimental session that consisted of two calibration phases and one copy spelling phase. Participants were seated approximately $90 \mathrm{~cm}$ away from a computer monitor that displayed an $8 \times 9$ matrix of letters and numbers. The speller matrix was adapted from a BCI2000 system used in a patient's home. After the participant was fitted for the electrode cap, an 8x9 matrix of letters and characters was presented on the computer monitor. For the calibration phase, participants were asked to focus their attention on a specific character in the matrix and to count how many times the letter flashes, while ignoring the images flashing over the other characters in the matrix. For example, as shown in Figure 7, the top left side of the display would show a word (e.g., WORDS) and the letter they should attend to is shown in parentheses at the end of the word. After a predetermined amount of flashes of each character (in this case 14) the matrix would stop flashing and after a 4-second pause the letter in parentheses would change to the next letter in the word (e.g., $(\mathrm{O}))$.

The session consisted of two calibration phases, counter-balanced, in which the participant made selections from a matrix presenting only the Face (Figure 7a) or only the White House (Figure 7b). Each participant spelled three six-letter words, 18 total characters, for each 
calibration. Following each calibration phase, a SWLDA analysis derived classification coefficients specific to each stimulus type.

Following calibration, participants completed an online copy-spelling task. The matrix presented the facial images over half of the matrix characters and the location images over the other half of the matrix characters (Figure 7c). Participants's selected 36 predetermined characters, 18 of the selections were facial stimuli and the facial classifier was used for online feedback, and 18 of the selections were location stimuli and the location classifier was used for online feedback. In the current version of the software it is not possible to use two classifiers simultaneously. Therefore, following all participants' sessions, offline analyses were conducted to examine how the facial classifier would perform when applied to the location data, and how the location classifier would perform when applied to the facial data. In a future implementation of the software the two classifiers will be used simultaneously. As mentioned previously, this will allow the system to rapidly eliminate half of the characters as potential target items.

\section{Experiment 2 Results}

\section{$\underline{\text { Statistical Analyses }}$}

A 2x2 Factorial Analysis of the Variance (ANOVA) was conducted to examine the effects of classifier type (Face or White House) and the effects of stimulus type (Face or White House) on offline analysis performance measures. Offline analyses were performed on predicted accuracy, target flashes, selections per minute, and bitrate. Offline accuracy is expressed as the predicted percentage of correctly selected characters for each word. Offline selections per minute are the predicted total character selections made correctly or incorrectly in a given minute.

Offline, predicted bitrate is calculated using the formula described by Pierce (1980). 


\section{Bitrate $=\log _{2} N+P+(1-P) \log _{2}(1-P / N-1)$}

Bitrate takes into account the number of possible targets $(\mathrm{N})$ and the probability that the target is classified accurately $(\mathrm{P})$. Dividing Bitrate by the trial duration in minutes results in bits per minute.

Paired sampled t-tests were used to examine the differences between the waveforms produced by each participant. Waveform analyses were conducted on the calibration data acquired for the Face only and White House only conditions (Figure 12).

\section{Offline Performance}

Predicted BCI performance accuracy produced by each classifier type applied to each stimulus type are shown in Figure 8. Analyses did not indicate a significant main effect of classifier type, $(F(1,9)=1.395, p=.268)$, or stimulus type $(F(1,9)=1.598, p=.238)$. In addition, the classifier type by stimulus type interaction was not significant $(\mathrm{F}(1,9)=1.598, \mathrm{p}=.238)$. 


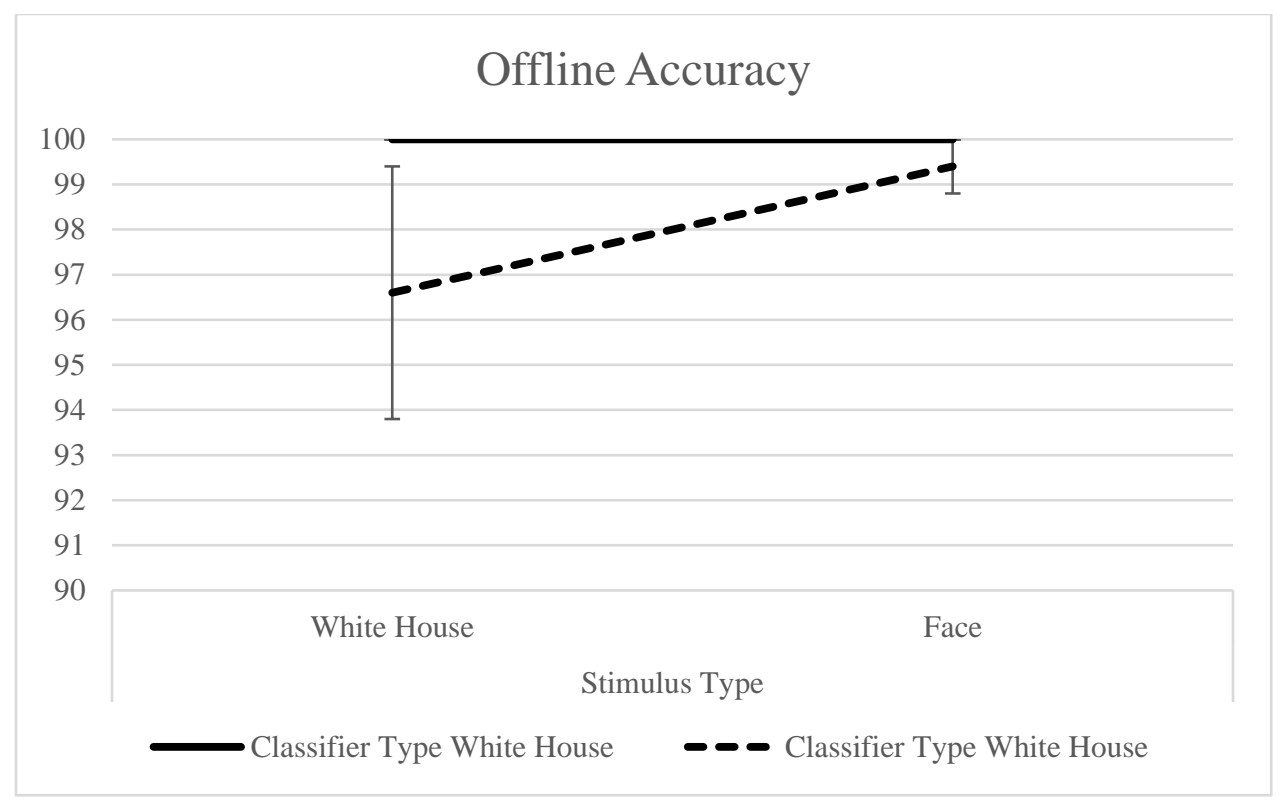

Figure 8. Experiment 2 offline accuracy: Predicted accuracy of each classifier type, White House classifier (solid black line) and Face classifier (dashed black line), when applied to each stimulus type.

Mean target flashes produced by each classifier type applied to each stimulus type are shown in Figure 9. Analyses did not indicate a significant main effect of classifier type $(\mathrm{F}(1,9)=.000, \mathrm{p}=1)$, or stimulus type $(\mathrm{F}(1,9)=1.823, \mathrm{p}=.210)$ Analyses, however, indicated a significant interaction of classifier type by stimulus type $(F(1,9)=17.053, p=.003)$. Pairwise comparisons indicated no significant differences between groups. 


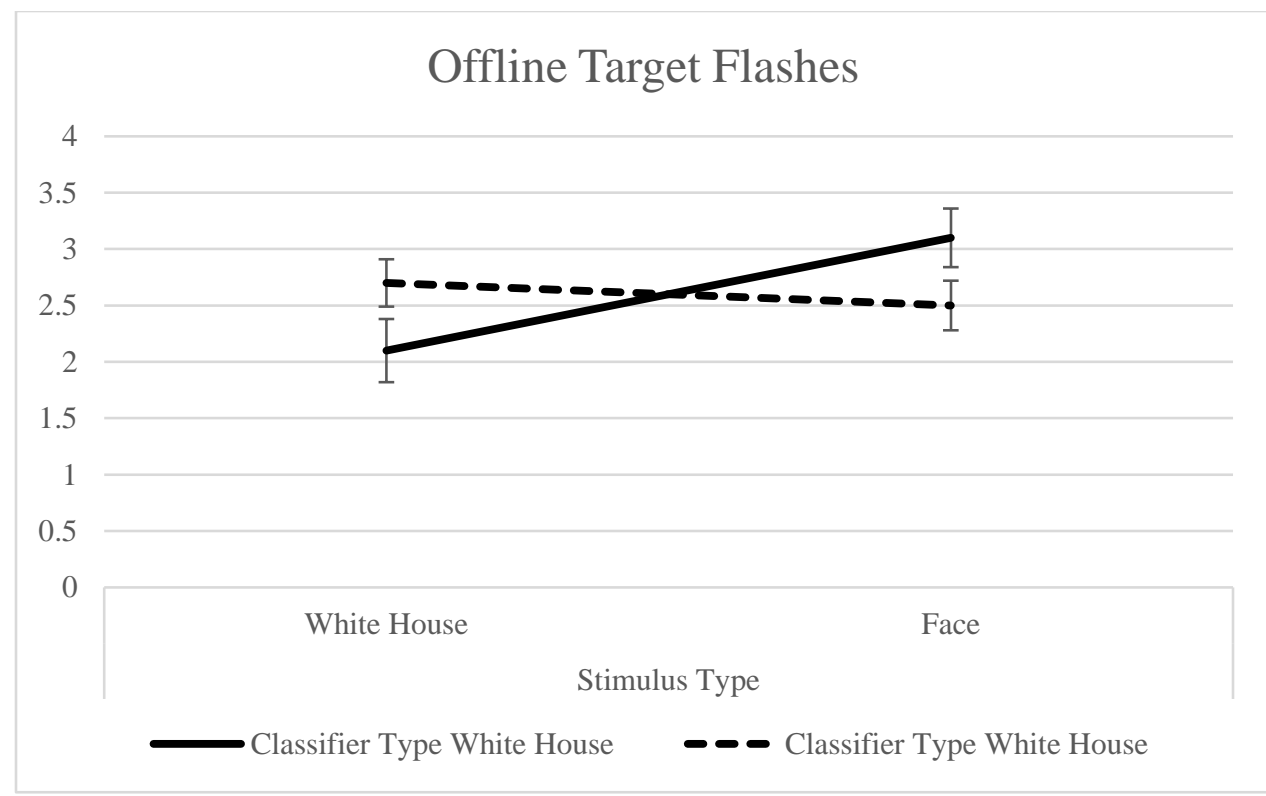

Figure 9. Experiment 2 offline target flashes: Predicted target flashes of each classifier type, White House classifier (solid black line) and Face classifier (dashed black line), when applied to each stimulus type.

Mean selections per minute produced by each classifier type applied to each stimulus type are shown in Figure 10. Analyses did not indicate a significant main effect either the classifier type $(\mathrm{F}(1,9)=.478, \mathrm{p}=.507)$, or stimulus type $(\mathrm{F}(1,9)=2.304, \mathrm{p}=.163)$. The analyses did indicate, however, a significant interaction of classifier type by stimulus type $(F(1,9)=12.886$, $\mathrm{p}=.006$ ) on selections per minute. Pairwise comparisons did not indicate significant differences between groups. 


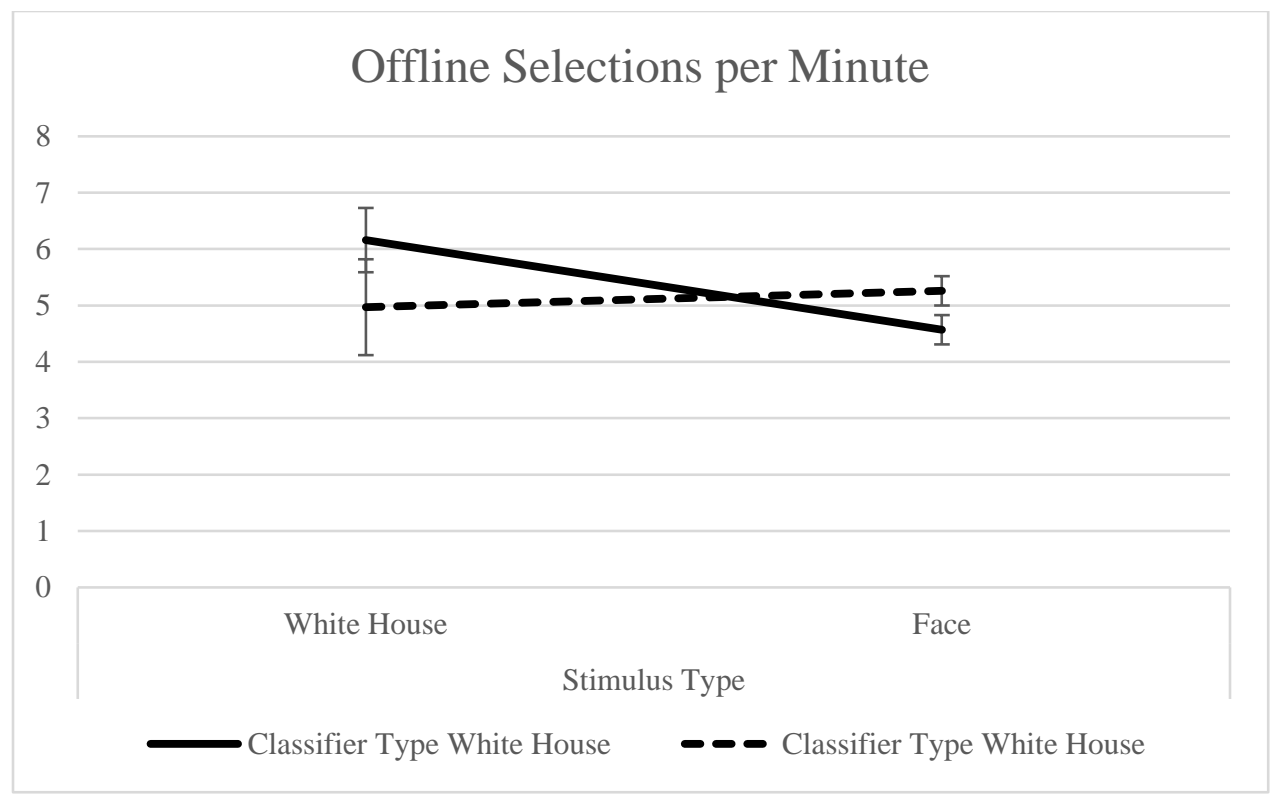

Figure 10. Experiment 2 offline selections per minute: Predicted selections per minute of each classifier type, White House classifier (solid black line) and Face classifier (dashed black line), when applied to each stimulus type.

Mean bitrates produced by each classifier type applied to each stimulus type are shown in Figure 11. Analyses did not indicate significant main effect either the classifier type $(\mathrm{F}(1,9)=1.312, \mathrm{p}=.282)$, or stimulus type $(\mathrm{F}(1,9)=1.458, \mathrm{p}=.258)$. The analyses did indicate significant interaction of classifier type by stimulus type $(F(1,9)=17.289, p=.002)$ on bitrate. Pairwise comparisons indicated no significant differences between groups. 


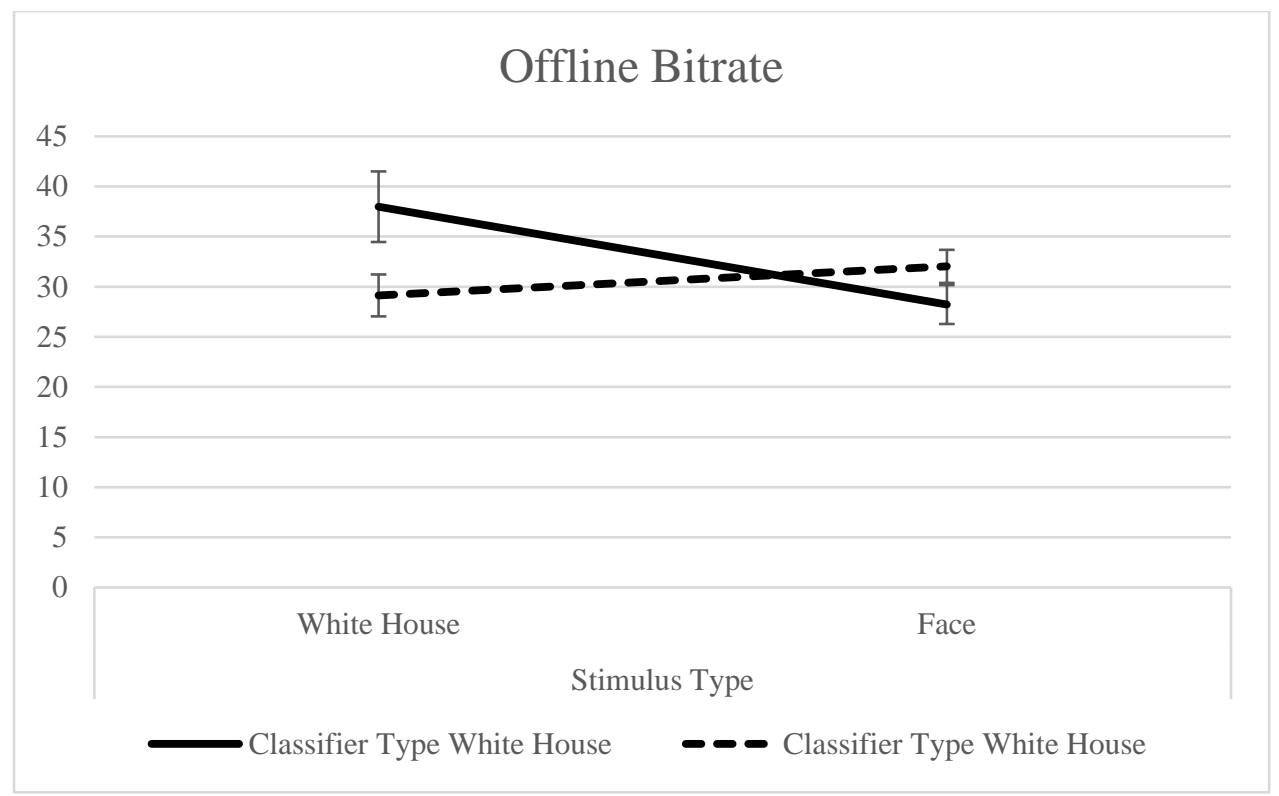

Figure 11. Offline bitrate: predicted bitrate of each classifier type, White House classifier (solid black line) and Face classifier (dashed black line), when applied to each stimulus type

$\underline{\text { Waveform Analysis }}$

The specified time windows examined for positive amplitudes and latencies were 150320ms and 350-550ms. The specified time windows to examine N170 and N400 amplitudes and latencies were sets to 128-195 for the N170 component, and 191-300 for the N400 component. Five electrode locations $\mathrm{Pz}, \mathrm{Cz}, \mathrm{PO} 7$, and PO8 were examined. 


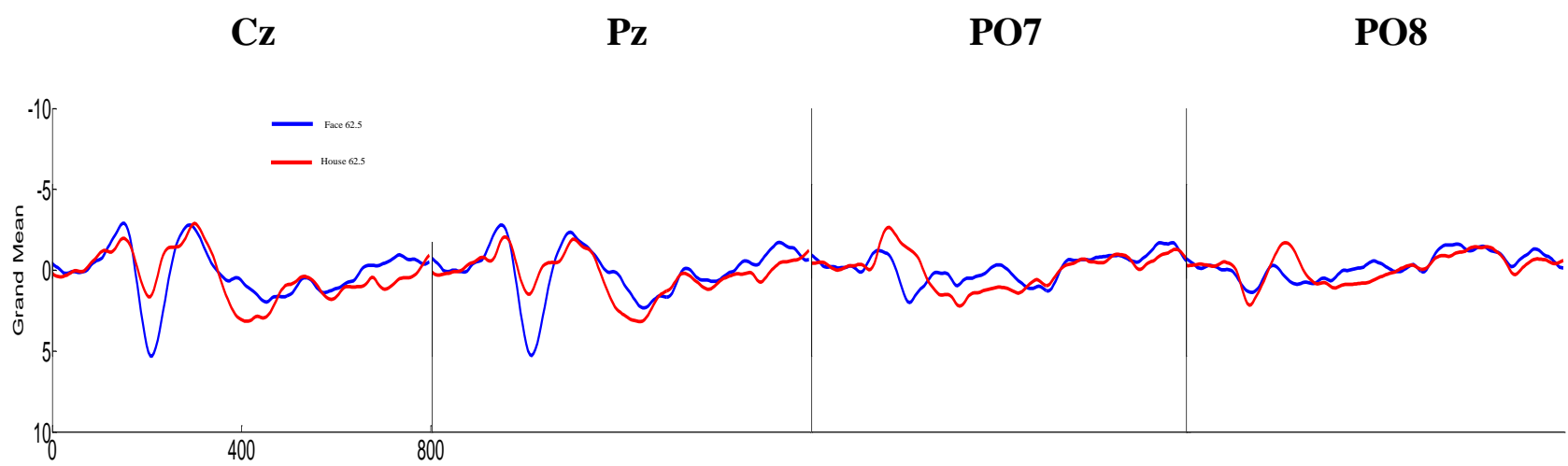

Figure 12. Grand mean waveforms for Experiment 2: Average waveforms for all ten participants for the two types of images, faces (blue line) and White House (red line) used in the BCI task for electrode locations Cz, Pz. PO7, and PO8.

Comparison of ERPs elicited by the Faces $(\mathrm{M}=5.97, \mathrm{SD}=1.26)$ and the White House $(\mathrm{M}=2.08, \mathrm{SD}=1.63)$ at electrode location $\mathrm{Pz}$ during time window $150-300 \mathrm{~ms}$ was shown to be significant $(\mathrm{t}(9)=7.642, \mathrm{p}<.001)$. Comparison of responses to the Faces $(\mathrm{M}=5.92, \mathrm{SD}=1.60)$ and the White House $(\mathrm{M}=2.26, \mathrm{SD}=1.21)$ at electrode location $\mathrm{Cz}$ during time window $150-300 \mathrm{~ms}$ was also shown to be significant $(\mathrm{t}(9)=6.059, \mathrm{p}<.001)$. No significant differences, however, were found for amplitudes during time window 150-300ms at electrode locations PO7 and PO8. Furthermore, t-tests indicated no significant differences in latencies produced by Faces or White House during 150-300ms at any of the four electrode locations that were examined.

Analyses indicated no significant differences between the amplitudes or the latencies produced by the Face and White House conditions during time window 350-550ms at any of the four electrode locations. In addition, analyses did not indicate significant differences between the amplitudes of latencies produced by either condition during time window 128-195ms at any of the four electrode locations. 
Analyses did indicate a significant difference between the amplitudes produced by the Face $(\mathrm{M}=-1.49, \mathrm{SD}=.89)$ and White House $(\mathrm{M}=-2.46, \mathrm{SD}=1.26)$ conditions, during time window $191-300 \mathrm{~ms}$ at electrode location PO8 only $(\mathrm{t}(9)=3.277, \mathrm{p}=.01)$. The remaining analyses of amplitudes and latencies during time window 191-300ms did not indicate significant differences between the two conditions at the other three electrode locations.

\section{Experiment 2 Discussion}

Offline analyses were used to provide evidence that a classifier created for a specific stimulus could result in better BCI performance. Despite no significant differences, offline accuracy predicted sufficiently high accuracy for each classifier type applied to each stimulus type. Due to the use of able-bodied undergraduates as a sample, high accuracies were anticipated and a ceiling effect may have prevented our ability to detect significant differences in accuracy. Unexpectedly, however, the White House classifier predicted the highest accuracy when applied to both the White House stimulus and Face stimulus.

The predicted target flashes indicated that classifiers applied to the corresponding stimulus would require significantly fewer target flashes to make an accurate character selection. The White House classifier applied to the White House stimulus and the Face classifier applied to the Face stimulus required the least amount of target flashes required to make a character selection. Predicted selections per minute indicated that classifiers applied to the corresponding stimulus could influence the amount of character selections made per minute. The White House classifier applied to the White House stimulus and the Face classifier applied to the Face stimulus predicted the highest number of selections per minute, as well as the highest bitrate. 
The ERPs produced by the two stimuli differed in the amplitudes at the first positive window for electrodes $\mathrm{Pz}$ and $\mathrm{Cz}$. The only significant difference in negative amplitude was at the second negative time window at electrode location PO8. Based on our findings, it appears that the BCI system can discriminate between the ERPs produced by each stimulus type. Offline performance measures indicate that having a specific classifier for specific stimuli in the matrix could result in increased BCI performance on levels of target flashes, selections per minute, and bitrate. 


\section{CHAPTER 4}

\section{EXPERIMENT 3}

Oddball data was collected to examine whether face stimuli and location stimuli would produce significantly different ERPs. This was to provide a rationale for using these specific stimulus types, the Einstein face and the White House location images, for Experiment 2. Differences between the N170 and N400 components, often associated with facial processing, were expected but not found. The two different stimuli did appear to produce differing P300 responses.

Previous research has shown that the use of two-stimulus paradigms may increase BCI performance (Kaufmann et al., 2014). In contrast to previous research, Experiment 2 examined the use of two types of stimuli, facial and location, which have been shown to produce distinct ERPs. This was done with the intent to provide a rationale for the development of classifiers that identify specific stimuli in a two-stimulus matrix presentation. To further investigate the use of two-stimulus paradigms, Experiment 3 examined a third type of stimuli (i.e. graspable objects) to determine if they would produce distinctly different ERPs from location stimuli.

\section{Graspable Objects as Stimuli: Tools}

Based on the offline performance analysis of Experiment 2, the use of facial and location stimuli were sufficient stimuli to provide evidence for the use of a two-stimulus, two-classifier paradigm. The main hypothesis was that these stimuli would produce significantly different N170 and N400 components. However, this result was not observed. The observed differences were in the P300 component. The rationale behind using facial stimuli was based on previous 
EEG studies, as well as neuroimaging studies that identified the FFA. The location stimuli were chosen due to neuroimaging indicating distinct activation in the PPA.

Functional MRI studies can discriminate the FFA from the PPA due to the high spatial resolution that MRI produces. In our study, we hypothesized that these differences would also be observed in the scalp recorded EEG. We expect this result was not observed due to the close proximity of the PPA and FFA. The PPA is located at the medial portion of the fusiform gyrus, whereas the FFA is located at a nearby cortical region in the mid-fusiform gyrus. Therefore, Experiment 3 examined another possible stimulus, images of tools, which activate more frontal areas such as the premotor and motor cortex.

Neuroimaging studies have shown a unique cognitive response to graspable objects such as tools (Creem-Regehr, \& Lee, 2005). Tools are considered a unique class of objects, due to the relationship between object recognition as well as the potential actions that can be performed with the object (Handy, Grafton, Shroff, Ketay, \& Gazzaniga, 2003). Viewing images of tools has been shown to activate the premotor cortex, and research has suggested that the priming of visual systems by viewing tools also primes motor systems (Grafton, Fadiga, Arbib, \& Rizzolatti, 1997; Tucker \& Ellis, 2004).

Experiment 2 examined the two-stimulus, two-classifier paradigm with facial (Face) and location (White House) stimuli, and results indicated that the White House classifier when applied to the White House stimulus and the Face classifier applied to the Face stimulus resulted in significantly less target flashes, greater selections per minute, and higher bitrate compared to each classifier applied to the non-corresponding stimulus. Because of the slightly higher performance produced by the location stimuli for our sample, Experiment 3 compared location 
stimuli and tool stimuli to examine whether tool stimuli could produce a more distinct ERP response from the location stimuli, thus further supporting that the two-stimulus paradigm could impact BCI performance.

\section{Experiment 3 Methods}

\section{$\underline{\text { Participants }}$}

Twenty-four able-bodied participants (10 men, 14 women; age range 19-42) were recruited from East Tennessee State University. Seven of the participants had prior BCI experience, the remainder of participants were naïve to BCI use. Sample size was determined using a Bayes factor (Rouder, Speckman, Sun, Morey, \& Iverson, 2009) stopping criterion of $\mathrm{BF}_{01}=30$. The study was approved by the East Tennessee State Institutional Review Board and each participant gave informed consent.

\section{Data Acquisition and Processing}

Data acquisition and processing as described in Experiment 2 was also used for Experiment 3.

\section{$\underline{\text { Classification }}$}

Stepwise Linear Discriminate Analysis (SWLDA) as described in the Experiment 2 was also used for Experiment 3.

\section{Experimental Stimuli, Procedure, and Design}

Participants completed one experimental session consisting of two calibration phases and one copy-spelling phase. Participants were seated approximately $90 \mathrm{~cm}$ away from a computer monitor that displayed an $8 \times 9$ matrix of letters and numbers. The speller matrix was adapted 
from a BCI2000 system used in a patient's home. After the participant was fitted for the electrode cap, an 8x9 matrix of letters and characters was presented on the computer monitor. For the White House calibration phase, participants were asked to focus their attention on a specific character in the matrix and to count how many times the image appeared, while ignoring the images flashing over the other characters in the matrix. For example, as shown in Figure 7, the top left side of the display would show a word (e.g., WORDS) and the letter they should attend to is shown in parentheses at the end of the word. After a predetermined amount of flashes of each character (in this case 10) the matrix would stop flashing and after a 4-second pause the letter in parentheses would change to the next letter in the word (e.g., $(\mathrm{O}))$. For the Tool calibration, participants were asked to perform a motor imagery task by focusing their attention on a specific character in the matrix and to imagine themselves using the object each time the image of the object flashes. This was done to elicit a stronger response in the premotor cortex than simply counting the number of character flashes. Following each calibration phase, a SWLDA analysis derived classification coefficients specific to each stimulus type.

Following calibration, participants completed an online copy-spelling task. The matrix presented the facial images over half of the matrix characters and the location images over the other half of the matrix characters (Figure 13). Participants's selected 36 predetermined characters, 18 of the selections were graspable object stimuli and the graspable object classifier was used for online feedback, and 18 of the selections were location stimuli and the location classifier was used for online feedback. In the current version of the software it is not possible to use two classifiers simultaneously. Therefore, following all participants' sessions, offline analyses were conducted to examine how the graspable object classifier would perform when applied to the location data, and how the location classifier would perform when applied to the 
graspable object data. In a future implementation of the software the two classifiers will be used simultaneously. As mentioned previously, this will allow the system to rapidly eliminate half of the characters as potential target items.
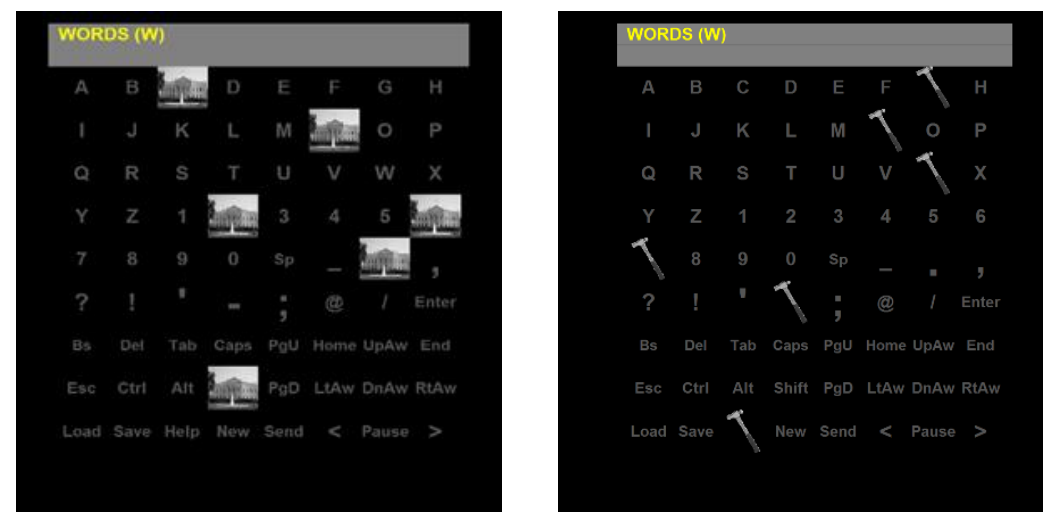

Figure 13. Example of one-stimulus matrices: Displaying the image of the White House only (left) and one-stimulus matrix displaying the image of the Tool only (right).

\section{Experiment 3 Results}

$\underline{\text { Statistical Analyses }}$

Repeated measures analysis of the variance (ANOVA) was used to examine the effects of classifier type (White House or Tool) and the effects of stimulus type (White House or Tool) on offline analysis performance measures. Offline analyses were performed on predicted accuracy, target flashes, selections per minute, and bitrate. Offline accuracy is expressed as the predicted percentage of correctly selected characters for each word. Offline selections per minute are the predicted total character selections made correctly or incorrectly in a given minute.

Offline, predicted bitrate is calculated using the formula described by Pierce (1980).

$$
\text { Bitrate }=\log _{2} N+P+(1-P) \log _{2}(1-P / N-1)
$$


Bitrate takes into account the number of possible targets $(\mathrm{N})$ and the probability that the target is classified accurately $(\mathrm{P})$. Dividing Bitrate by the trial duration in minutes results in bits per minute.

\section{$\underline{\text { Offline Performance }}$}

Repeated measures ANOVA examining offline accuracy produced by each classifier type applied to each stimulus type (Figure 14) found Mauchly's test of Sphericity to not be significant $(\mathrm{p}=.214)$ indicating no violation of the assumption of sphericity. With sphericity assumed, the ANOVA indicated significant differences between the four conditions $F(3,33)=8.42, \mathrm{p}<.001$. Post-hoc tests using Bonferroni corrections indicated the following significant differences: the comparison between accuracy for the White House classifier applied to White House stimuli $(\mathrm{M}=99.08, \mathrm{SD}=3.11)$ was significantly higher than the Tool classifier applied to White House stimuli ( $M=93.42, \mathrm{SD}=8.9), \mathrm{p}=.013$, and the White House classifier applied to Tool stimulus $(\mathrm{M}=89.79, \mathrm{SD}=14.5), \mathrm{p}=.013$. The Tool classifier applied to Tool stimuli $(\mathrm{M}=97.79, \mathrm{SD}=3.34)$ was significantly higher than the White House classifier applied to the Tool stimuli (M=89.79, $\mathrm{SD}=14.50), \mathrm{p}=.019$. 


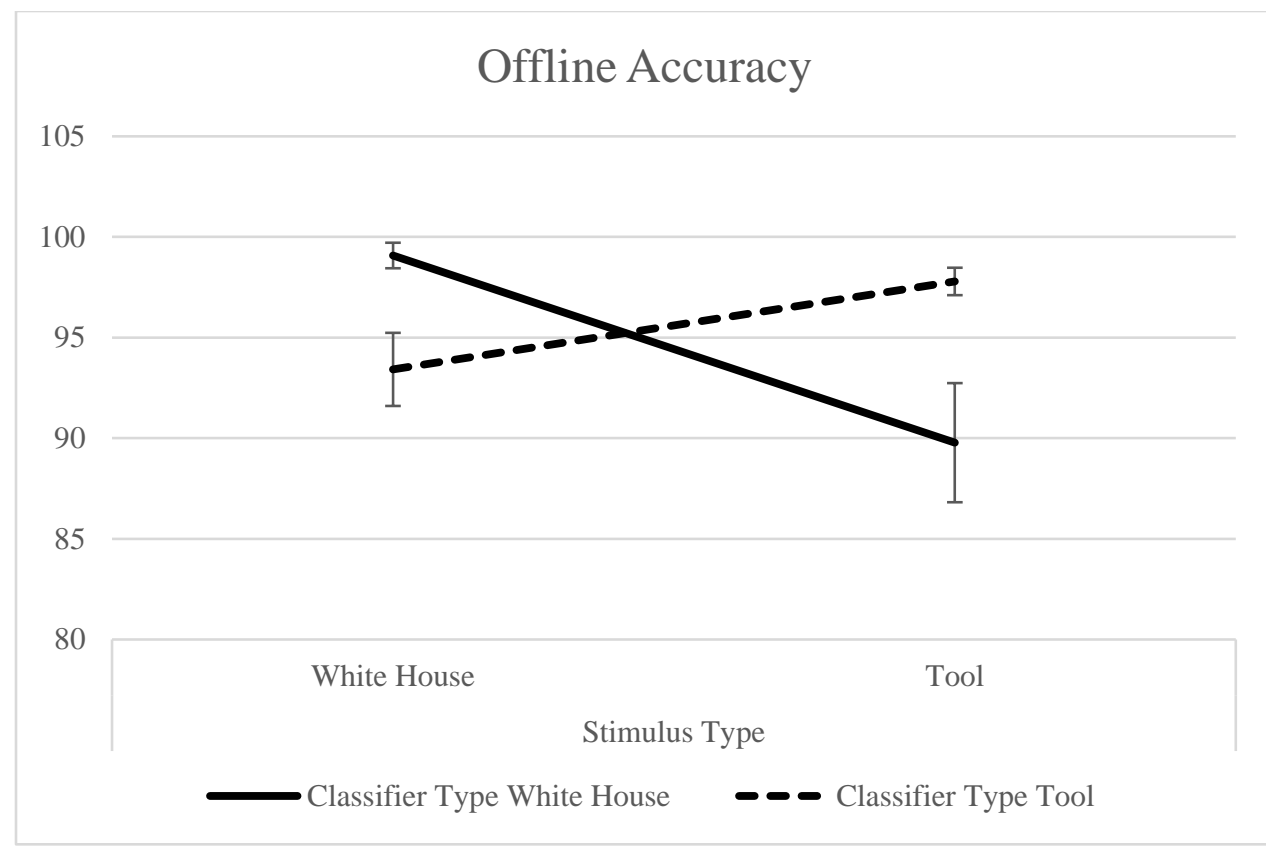

Figure 14. Experiment 3 offline accuracy: Predicted accuracy of each classifier type, White House classifier (solid black line) and Tool classifier (dashed black line), when applied to each stimulus type.

Repeated measures ANOVA examining offline target flashes produced by each classifier type applied to each stimulus type (Figure 15) found Mauchly's test of Sphericity to not be significant ( $\mathrm{p}=.674)$. With sphericity assumed, the ANOVA indicated significant differences between the four conditions $F(3,33)=22.21, \mathrm{p}<.001$. Post-hoc tests using Bonferroni corrections indicated the following significant differences: the comparison between target flashes for the White House classifier applied to White House Stimuli (M=2.58, SD=1.13) was significantly lower than the Tool classifier applied to White House stimuli ( $M=4.00, S D=1.10)$, $\mathrm{p}<.001$, and the White House classifier applied to Tool stimuli $(\mathrm{M}=4.04, \mathrm{SD}=.99), \mathrm{p}<.001$. The comparison between target flashes for the Tool classifier applied to Tool stimuli ( $\mathrm{M}=3.04$, 
$\mathrm{SD}=1.12$ ) was significantly lower than the Tool classifier applied to White House stimuli, $\mathrm{p}<.001$, and the White House classifier applied to Tool stimuli, $\mathrm{p}<.001$.

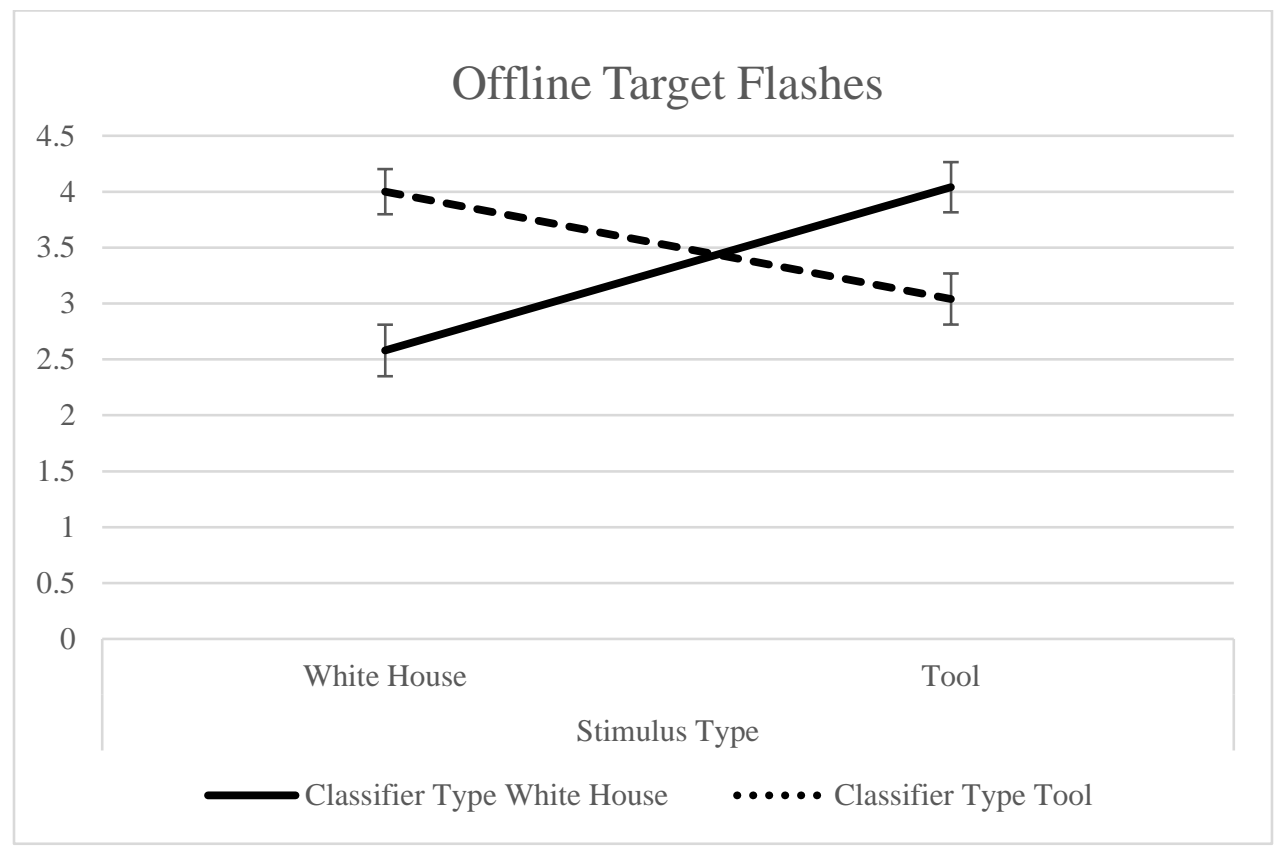

Figure 15. Experiment 3 Offline target flashes: Predicted target flashes of each classifier type, White House classifier (solid black line) and Face classifier (dashed black line), when applied to each stimulus type.

Repeated measures ANOVA examining offline selections per minute produced by each classifier type applied to each stimulus type (Figure 16) found Mauchly's test of Sphericity to not be significant $(\mathrm{p}=.326)$. With sphericity assumed, the ANOVA indicated significant differences between the four conditions $F(3,33)=19.93, \mathrm{p}<.001$. Post-hoc tests using Bonferroni corrections indicated the following significant differences: the comparison between the White House classifier applied to White House stimuli $(\mathrm{M}=5.46, \mathrm{SD}=1.7)$ was significantly higher than the Tool classifier applied to White House stimuli $(\mathrm{M}=3.83, \mathrm{SD}=.98), \mathrm{p}<.001$, and the White House classifier applied to Tool stimulus $(\mathrm{M}=3.74, \mathrm{SD}=.80), \mathrm{p}<.001$. Comparison 
between the Tool classifier applied to Tool stimulus $(\mathrm{M}=4.74, \mathrm{SD}=1.13)$ was significantly higher than the Tool classifier applied to White House stimulus, $\mathrm{p}=.001$, and the White House classifier applied to Tool stimuli, $\mathrm{p}<.001$.

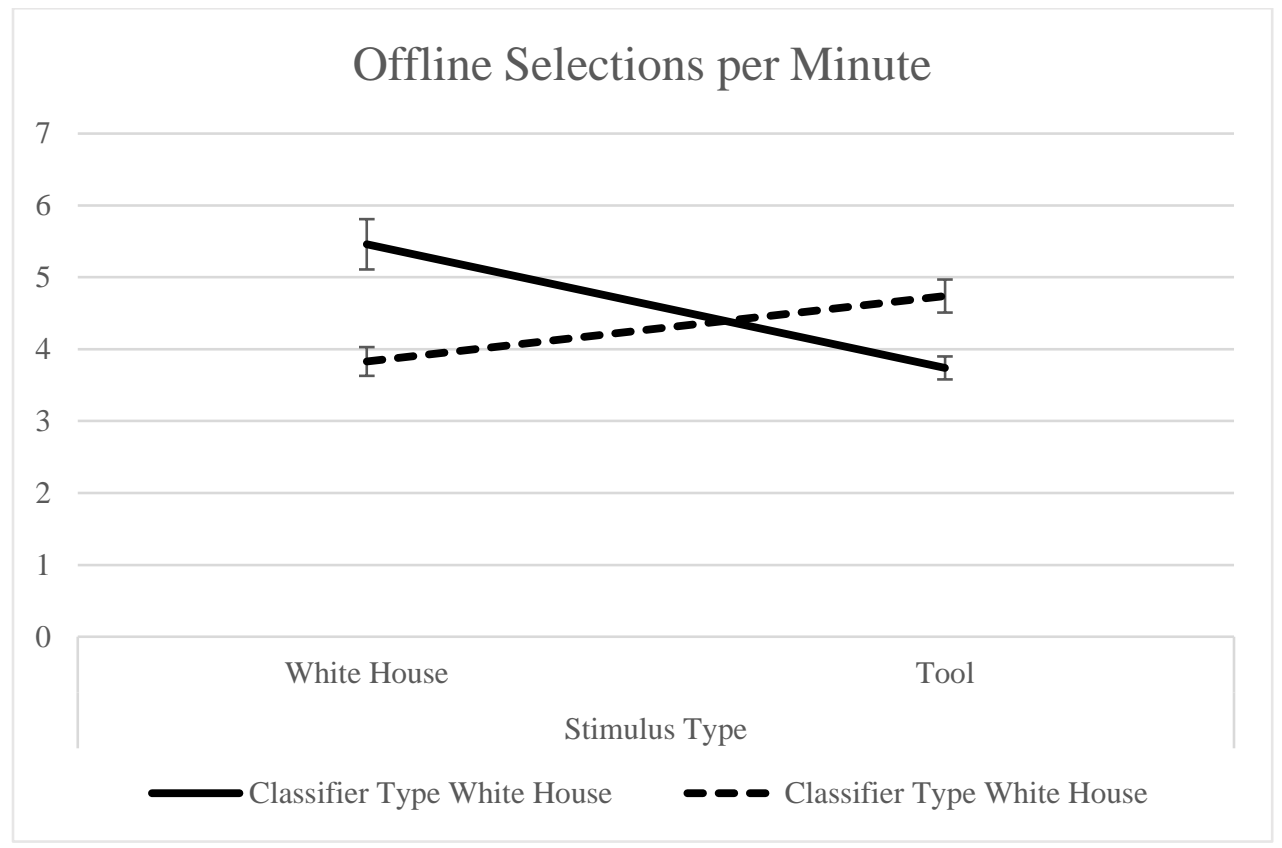

Figure 16. Experiment 3 offline selections per minute: Predicted selections per minute of each classifier type, White House classifier (solid black line) and Face classifier (dashed black line), when applied to each stimulus type.

Repeated measures ANOVA examining offline bitrate produced by each classifier type applied to each stimulus type (Figure 17) found Mauchly's test of Sphericity to not be significant $(\mathrm{p}=.314)$. With sphericity assumed, the ANOVA indicated significant differences between the four conditions $F(3,33)=26.094, \mathrm{p}<.001$. Post-hoc tests using Bonferroni corrections indicated the following significant differences: the White House classifier applied to White House stimuli $(\mathrm{M}=33.39, \mathrm{SD}=11.02)$ was significantly higher than the Tool classifier applied to White House stimuli ( $\mathrm{M}=21.53, \mathrm{SD}=7.87), \mathrm{p}<.001$, and the White House classifier applied to Tool stimuli 
(20.07, $\mathrm{SD}=7.67), \mathrm{p}<.001$. The comparison between the Tool classifier applied to Tool stimulus $(\mathrm{M}=28.30, \mathrm{SD}=7.88$ ) was significantly higher than the Tool classifier applied to White House stimulus, $\mathrm{p}<.001$, and the White House classifier applied to Tool stimulus, $\mathrm{p}<.001$.

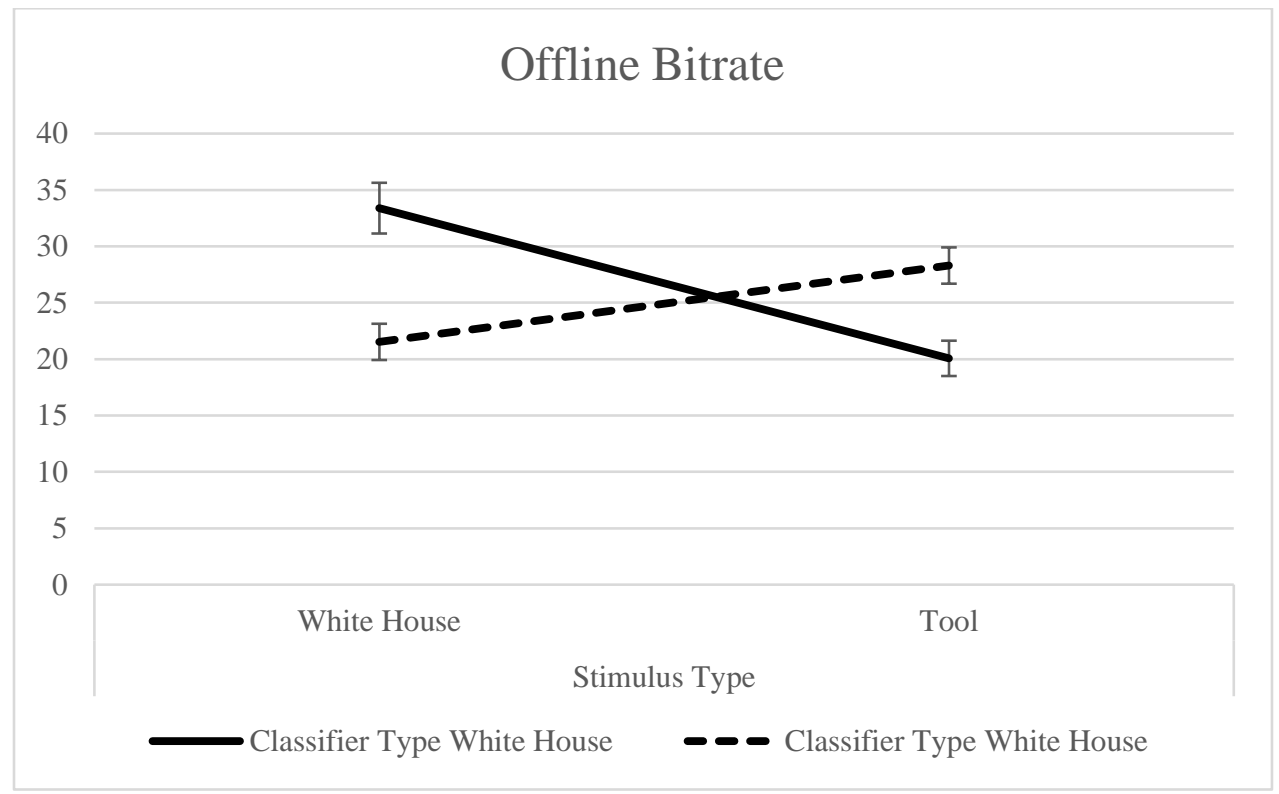

Figure 17: Experiment 3 offline bitrate: Predicted bitrate of each classifier type, White House classifier (solid black line) and Face classifier (dashed black line), when applied to each stimulus type.

$\underline{\text { Waveform Analysis }}$

The specified time windows examined for positive amplitudes and latencies were 150$320 \mathrm{~ms}$ and $350-550 \mathrm{~ms}$. The specified time windows to examine N170 and N400 amplitudes and latencies were sets to 128-195 for the N170 component, and 191-300 for the N400 component. Eight electrode locations Pz, Cz, PO7, PO8, F3, F4, FC5, and FC6 were examined (Figure 18). Paired samples t-tests were used to compare differences between the two types of stimuli. 


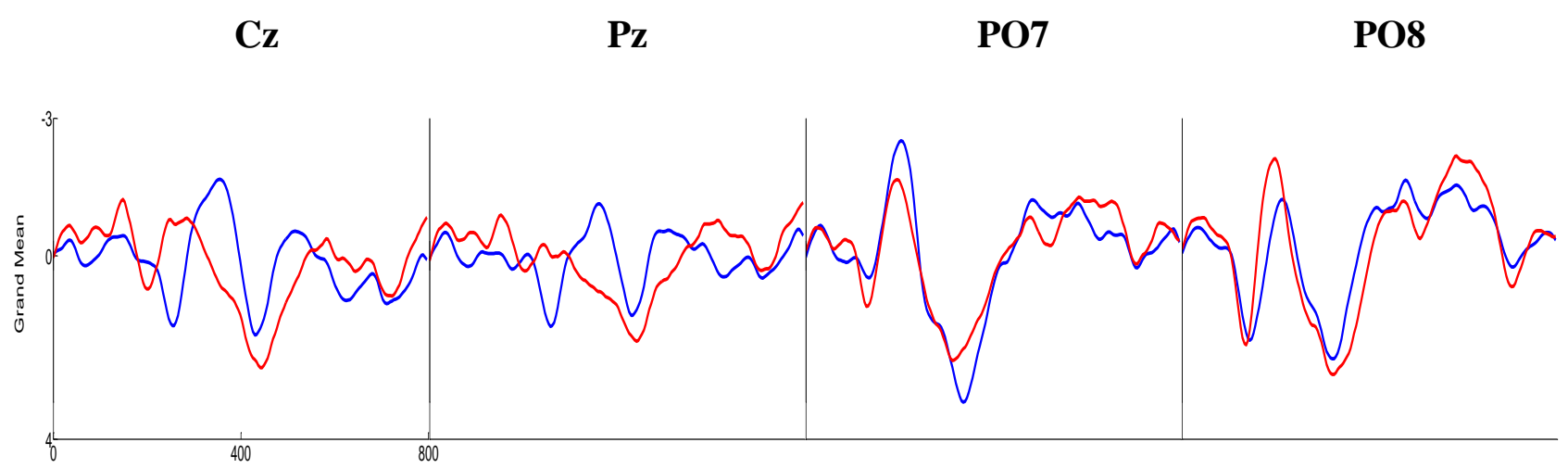

F3

F4

FC5

FC6

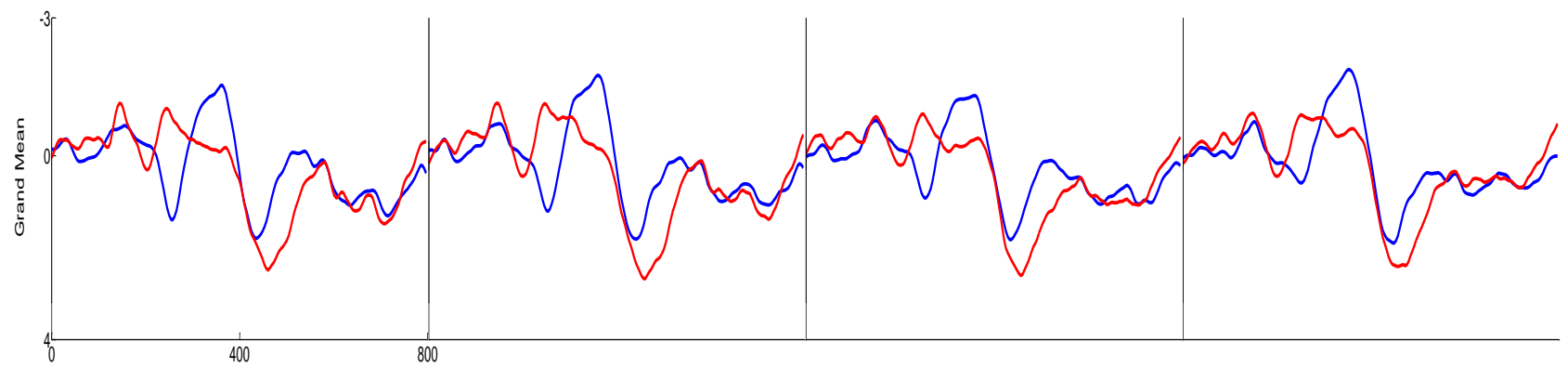

Figure 18. Grand mean waveforms for Experiment 3: Average waveforms for all 24 participants for the two types of images, Tool (blue line) and White House (red line) used in the BCI task for electrode locations Cz, Pz. PO7, PO8, F3, F4, FC5, and FC6.

Comparison of amplitudes of Tool $(\mathrm{M}=2.58, \mathrm{SD}=1.02)$ and White House $(\mathrm{M}=3.38$, $\mathrm{SD}=1.79$ ) at electrode location PO8 during positive time window $150-300 \mathrm{~ms}$ was shown to be significant, $t(23)=-2.716, p=.012$. No significant differences were found for amplitudes during time window 150-300ms at electrode locations Pz, Cz, PO7, F3, F4, FC5, and FC6.

Comparison of latencies of Tool $(\mathrm{M}=200.03, \mathrm{SD}=60.32)$ and White House $(\mathrm{M}=249.51$, $\mathrm{SD}=47.83$ ) at electrode location PO8 during time window 150-300ms was shown to be significant, $\mathrm{t}(23)=-2.898, \mathrm{p}=.008$. Comparison of latencies of Tool $(\mathrm{M}=248.86, \mathrm{SD}=34.94)$ and White House ( $\mathrm{M}=228.51, \mathrm{SD}=40.1)$ at electrode location FC6 during time window 150-300ms 
was shown to be significant, $\mathrm{t}(23)=3.326, \mathrm{p}=.003$. No significant differences were found for latencies during time window 150-300ms at electrode locations Pz, Cz, PO7, F3, F4, and FC5.

Comparison of amplitudes of Tool $(\mathrm{M}=2.01, \mathrm{SD}=1.85)$ and White House $(\mathrm{M}=3.01$, $\mathrm{SD}=1.46$ ) at electrode location $\mathrm{Pz}$ during positive time window 350-550ms was shown to be significant, $\mathrm{t}(23)=-3.72, \mathrm{p}=.001$. Comparison of amplitudes of Tool $(\mathrm{M}=2.64, \mathrm{SD}=1.93)$ and White House $(\mathrm{M}=3.37, \mathrm{SD}=1.56)$ at electrode location $\mathrm{Cz}$ during positive time window 350$550 \mathrm{~ms}$ was shown to be significant, $\mathrm{t}(23)=-2.492, \mathrm{p}=.020$. Comparison of amplitudes of Tool $(\mathrm{M}=3.88, \mathrm{SD}=2.62)$ and White House $(\mathrm{M}=2.83, \mathrm{SD}=1.78)$ at electrode location PO7 during positive time window $350-550 \mathrm{~ms}$ was shown to be significant, $\mathrm{t}(23)=2.385, \mathrm{p}=.026$. Comparison of amplitudes of Tool $(\mathrm{M}=2.00, \mathrm{SD}=1.26)$ and White House $(\mathrm{M}=2.76, \mathrm{SD}=1.54)$ at electrode location PO8 during positive time window 350-550ms was shown to be significant, $\mathrm{t}(23)=-2.168$, $\mathrm{p}=.041$. Comparison of amplitudes of Tool $(\mathrm{M}=3.36, \mathrm{SD}=2.13)$ and White House $(\mathrm{M}=4.51$, $\mathrm{SD}=2.77$ ) at electrode location $\mathrm{F} 3$ during positive time window 350-550ms was shown to be significant, $\mathrm{t}(23)=-2.931, \mathrm{p}=.008$. Comparison of amplitudes of Tool $(\mathrm{M}=3.47, \mathrm{SD}=4.67)$ and White House $(M=4.67, S D=3.11)$ at electrode location F4 during positive time window 350$550 \mathrm{~ms}$ was shown to be significant, $\mathrm{t}(23)=-3.570, \mathrm{p}=.002$. Comparison of amplitudes of Tool $(\mathrm{M}=2.88, \mathrm{SD}=1.64)$ and White House $(\mathrm{M}=3.76, \mathrm{SD}=1.71)$ at electrode location FC5 during positive time window $350-550 \mathrm{~ms}$ was shown to be significant, $\mathrm{t}(23)=-3.060, \mathrm{p}=.006$.

Comparison of amplitudes of Tool $(\mathrm{M}=2.95, \mathrm{SD}=1.52)$ and White House $(\mathrm{M}=3.65, \mathrm{SD}=1.87)$ at electrode location FC6 during positive time window 350-550ms was shown to be significant, $\mathrm{t}(23)=-3.807, \mathrm{p}=.001$. Analyses did not indicate significant differences between the latencies produced by the conditions during time window 350-550ms at any of the eight electrode locations. 
Comparison of amplitudes of Tool $(\mathrm{M}=-0.72, \mathrm{SD}=1.48)$ and White House $(\mathrm{M}=-2.18$, $\mathrm{SD}=2.06$ ) at electrode location PO8 during the first negative time window 128-195ms was shown to be significant, $t(23)=3.251, p=.004$. No significant differences were found for amplitudes during negative time window 128-195ms at electrode locations Pz, Cz, PO7, F3, F4, and FC5. Comparison of latencies of Tool $(\mathrm{M}=173.82, \mathrm{SD}=27.01)$ and White House $(\mathrm{M}=188.31$, $\mathrm{SD}=15.49$ ) at electrode location P07 during the first negative time window 128-195ms was shown to be significant, $\mathrm{t}(23)=-2.395, \mathrm{p}=.025$. No significant differences were found for amplitudes during negative time window 128-195ms at electrode locations Pz, Cz, PO8, F3, F4, FC5, and FC6.

Comparison of amplitudes of Tool $(\mathrm{M}=-0.73, \mathrm{SD}=.98)$ and White House $(\mathrm{M}=-2.01$, $\mathrm{SD}=1.25$ ) at electrode location $\mathrm{Pz}$ during the second negative time window 191-300ms was shown to be significant, $\mathrm{t}(23)=5.234, \mathrm{p}<.001$. Comparison of amplitudes of Tool $(\mathrm{M}=-1.74$, $\mathrm{SD}=.97)$ and White House $(\mathrm{M}=-2.22, \mathrm{SD}=1.27)$ at electrode location $\mathrm{Cz}$ during the second negative time window $191-300 \mathrm{~ms}$ was shown to be significant, $\mathrm{t}(23)=2.348, \mathrm{p}=.028$. Comparison of amplitudes of Tool $(\mathrm{M}=-1.72, \mathrm{SD}=1.48)$ and White House $(\mathrm{M}=-2.87, \mathrm{SD}=1.86)$ at electrode location PO7 during negative time window 191-300ms was shown to be significant, $\mathrm{t}(23)=3.886$, $\mathrm{p}=.001$. Comparison of amplitudes of Tool $(\mathrm{M}=-1.59, \mathrm{SD}=0.88)$ and White House $(\mathrm{M}=-2.6$, $\mathrm{SD}=1.84$ ) at electrode location F3 during negative time window 191-300ms was shown to be significant, $\mathrm{t}(23)=2.939, \mathrm{p}=.007$. Comparison of amplitudes of Tool $(\mathrm{M}=-1.51, \mathrm{SD}=0.76)$ and White House $(\mathrm{M}=-2.61, \mathrm{SD}=1.79)$ at electrode location F4 during negative time window 191$300 \mathrm{~ms}$ was shown to be significant, $\mathrm{t}(23)=3.043, \mathrm{p}=.006$. Comparison of amplitudes of Tool $(\mathrm{M}=-1.41, \mathrm{SD}=0.85)$ and White House $(\mathrm{M}=-2.17, \mathrm{SD}=1.29)$ at electrode location FC5 during negative time window $191-300 \mathrm{~ms}$ was shown to be significant, $\mathrm{t}(23)=2.365, \mathrm{p}=.027$. Comparison 
of amplitudes of Tool $(\mathrm{M}=-1.25, \mathrm{SD}=0.72)$ and White House $(\mathrm{M}=-1.99, \mathrm{SD}=1.35)$ at electrode location FC6 during negative time window 191-300ms was shown to be significant, $\mathrm{t}(23)=2.680$, $\mathrm{p}=.013$. Analyses did not indicate significant difference in amplitude at electrode location PO8. Analyses also did not indicate significant differences between the latencies produced by the conditions during time window $128-195 \mathrm{~ms}$ at any of the eight electrode locations.

\section{Experiment 3 Discussion}

In Experiment 3, offline analyses were used to provide evidence that a classifier created for a specific stimulus could result in better BCI performance in areas of accuracy, selections per minute, and bitrate in a two-stimulus presentation paradigm. Offline analyses of accuracy, selections per minute, and bitrate indicated significantly higher accuracy, selections per minute, and bitrate for the White House classifier applied to White House stimulus and for the Tool classifier applied to Tool stimulus than classifiers applied to the non-corresponding stimulus. These findings support our hypothesis that a stimulus specific classifier when applied to the corresponding stimulus can result in increases in BCI performance.

Functional MRI research indicating activation in the parahippocampal place area in response to visual processing of location stimuli (Agguire et al., 1998; Epstein et al., 1999; Malach et al., 2002) as well as activation in the premotor cortex in response to stimuli such as graspable objects (Creem-Regehr, \& Lee, 2005; Grafton et al.,1997; Tucker \& Ellis, 2004;) prompted the examination of parietal locations (PO7 and PO8) and frontal locations (F3, F4, FC5, and FC6) in addition to locations Pz an Cz. Due to lack of EEG research on the ERPs produced by these stimulus types, exploratory analyses were conducted. Higher amplitudes produced by the White House image were expected at parietal locations and higher amplitudes 
produced by the Tool image were expected at frontal locations. Waveform analyses comparing the ERPs produced by the two stimulus types showed higher amplitude produced by the White House image than the Tool image at each of the examined electrode locations, except for PO7 in positive time window 350-550ms. This finding was unexpected, as higher amplitude for the Tool image was expected at frontal locations due to electrode proximity to the premotor cortex.

The findings of the offline performance measures indicating slightly higher performance in our sample for the White House classifier applied to White House stimulus was supported by the higher amplitudes found for the White House image at most of the electrode locations at the examined time points. The White House image appears to have produced the most robust cognitive response, possibly resulting in better target discrimination as depicted in offline predicted accuracy, selections per minute, and bitrate for our sample. The findings of Experiment 3 demonstrate that different types of stimuli could be used to add additional information to the classification algorithm. These findings offer support for the use of two types of stimuli to elicit different ERPs to be detected by a classifier. With this information, future research will develop stimulus specific classifiers to be tested online in a two-stimulus presentation paradigm. With the development of two stimulus specific classifiers, when the ERPs elicited by one of the two different stimuli, the system should be able to eliminate half of the characters in the matrix as potential targets. Thus, decreasing the number of potential selection errors. 


\section{CHAPTER 5}

\section{CONCLUSION}

P300 BCI technology has shown to be an effective method of communication, however, due to the relatively slow rate of communication there are many improvements to be made. The first online P300-based BCI study resulted in accuracy of 35 percent (Donchin, Spencer, \& Wijesinghe, 2000). Since this time, online accuracy is consistently near 100 percent and provides a rate of communication up to eight character selections per minute. Although a great deal of progress has been made, further improvements in speed and accuracy are needed for the technology to rival assistive communication devices that rely on muscle movement. Therefore, techniques to ensure highly accurate and fast classification are important.

Recent studies have shown that ERP components associated with facial stimuli can improve BCI performance in a two-stimulus presentation paradigm (Kaufmann et al., 2014). The present work extends these findings and incorporated two additional types of novel stimuli, location and graspable objects. Prior to this study, ERPs produced by location and graspable objects have not been examined; however, fMRI data has provided evidence that these stimuli activate different brain regions. Thus, the purpose of the present study was to determine if location and graspable objects produce differential ERPs that can subsequently lead to an improvement in BCI speed and accuracy.

Experiment 2, using facial and location stimuli, showed no differences in face specific components; nonetheless, other ERP differences were observed and the location stimuli produced slightly better performance than facial stimuli. Kaufmann et al. (2011) first examine facial stimuli in able-bodied subjects, based on their positive results, they extended the paradigm 
to people with severe speech and communication disorders and confirmed that facial stimuli produced higher speed and accuracy in this population as well. The findings of our project indicate that location stimuli produce comparable performance to facial stimuli. Extrapolating the results of the current study, we suggest that location stimuli may be beneficial for people with severe speech and communication disorders. This hypothesis should be tested in future studies.

In Experiment 3, location stimuli were compared to graspable object stimuli. As with Experiment 2, differential ERPs were observed for the two classes of stimuli and performance was once again higher is the location stimulus condition. These results support our hypothesis that unique classifiers may provide higher performance in a two-stimulus paradigm, as compared to the current methodologies that rely on a single classifier.

As with most P300 BCI research conducted in laboratory settings, an inherent limitation to our study design is the use of able-bodied participants as our sample. The goal of the current study, however, was to provide proof of concept using offline analyses. Nonetheless, due to the early stages of this line of research, it would not be appropriate to test the current paradigm with people who have severe speech and physical impairments. Another limitation of the study design is that it did not afford us the opportunity to compare graspable object stimuli to facial stimuli. Further investigation comparing graspable object stimuli to facial stimuli may be beneficial to determine which would be more useful in the two-stimulus paradigm. Overall, evidence suggests that these three stimuli may be effective in a two-stimulus two-classifier paradigm, and these findings provide justification for progressing further in the development of such a paradigm. 


\section{REFERENCES}

Agguire, G.K., Zarahn, E., \& D’Esposito, M. (1998). An area within human ventral cortex sensitive to "building" stimuli: Evidence and implications. Neuron, 21, (2), 373-383.

Chen, L., Jin, J., Wang, X., \& Cichocki, A. (2015). A survey of the dummy face and human face stimuli used in BCI paradigm. Journal of Neuroscience Methods, (239), 18-27. http://dx.doi.org/10.1016/j.jneumeth.2014.10.002

Creem-Regehr, S.H., \& Lee, J.N. (2005). Neural representations of graspable objects: are tools special? Cognitive Brain Research, 22, 457-469. doi:10.1016/j.cogbrainres.2004.10.006

Colwell, K.A., Ryan, D.B., Throckmorton, C.S., Sellers, E.W., \& Collins, L.M. (2014). Channel selection methods for the P300 Speller. Journal of Neuroscience Methods, (232), 6-15.

Draper, N.R., \& Smith, H. (1981) Applied regression analysis. 2. New York: Wiley.

Donchin, E., Spencer, K.M., \& Wijesinghe, R. (2000). The mental prosthesis: Assessing the speed of a P300-based brain-computer interface. IEEE Transaction on Rehabilitation Engineering, 8(2), 174-179.

Donchin, E., \& Coles M.G.H. (1988) Is the P300 component a manifestation of context updating? Behavioral Brain Science, 11, 355-425.

Epstein, R., \& Kanwisher, N. (1998). A cortical representation of the local visual environment. Nature, 392, 598-601.

Epstein, R., Harris, A., Stanley, D., \& Kanwisher, N. (1999).The parahippocampal place area: Recognition, navigation, or encoding? Neuron, 23, 115-125. 
Fabiani, M., Gratton, G., Karis, D., \& Donchin, E. (1987). Definition, identification, and reliability of measurement of the P300 component of the event-related brain potential. Advances in Psychophysiology, 2, 1-78.

Farwell, L., \& Donchin, E. (1988). Talking off the top of your head: toward a mental prosthesis utilizing event-related brain potentials. Electroencephalography and Clinical Neurophysiology 70, 510-523. doi: 10.1016/0013-4694(88)90149-6

Grafton, S.T., Fadiga, L., Arbib, M.A., \& Rizzolatti, G. (1997). Premotor cortex activation during observation and naming of familiar tools. Neuroimage, 6, 231-236.

Handy, T.C., Grafton, S.T., Shroff, N.M., Ketay, S., \& Gazzaniga, M.S. (2003). Graspable objects grab attention when the potential for action is recognized. Nature Neuroscience, 6 (4), 421-427. doi:10.1038/nn1031.

Hillyard, S.A., \& Kutas, M. (1983). Electrophysiology of cognitive processing. Annual Review of Psychology, 34. 33-61. doi: 0066-4308/83/0201-0033\$02.00

Kanwisher, N., McDermott, J., \& Chun, M.M. (1997). The fusiform face Area: A module in human extrastiate cortex specialized for face perception. The Journal of Neuroscience, 17, (11), 4302-4311. doi: 0270-6474/97/174302-10\$05.00/0.

Kaufmann, T. \& Kubler., A. (2014). "Beyond maximum speed--a novel two-stimulus paradigm for brain-computer interfaces based on event-related potentials (P300-Bci)." Journal of Neural Engineering 11,(5). 056004. http://dx.doi.org/10.1077/1741-2560/11/5/056004

Kaufmann, T., Schulz, S. M., Koblitz, A., Renner, G., Wessig, C. \& Kubler, A. (2013). Face stimuli effectively prevent brain-computer interface inefficiency in patients with 
neurodegenerative disease. Clinical Neurophysiology, (124), 5. 893-900. http://dx.doi.org/10.1015/j.clinph.2012.11.006

Kaufmann, T., Schulz, S. M., Grunzinger, C., \& Kubl er, A. (2011). Flashing characters with famous faces improves ERP-based brain-computer interface performance. Journal of Neural Engineering (8), 5. 056016. http://dx.doi.org/10.1088/1741-2560/8/5/056016

Krusienski, D. J., Sellers, E. W., McFarland, D. J., Vaughn, T. M., \& Wolpaw, J. R. (2008). Toward enhanced P300 speller performance. Journal of Neuroscience Methods, (167), 15-21. http://dx.doi.org/10.1016/j.jneumeth.2007.07.017

Krusienski, D.J., Sellers, E.W., Cabestaing, F., Bayoudh, S., McFarland, D.J., Vaughan, T.M., \& Wolpaw, J.R. (2006). A comparison of classification techniques for the P300 Speller. Journal of Neural Engineering, (4), 3, 299-305. f

Kubler, A., Kotchoubey, B., Kaiser, J., Wolpaw, J. R., \& Birbaumer, N. (2001). Brain-computer communication: Unlocking the locked in. Psychological Bulletin, 127, (3), 358-75. http://dx.doi.org/10.1037//0033-2909.127.3.358

Jin, J., Daly, I., Zhang, Y., Wang, X., \& Cichocki, A. (2014). An optimized ERP brain-computer interface based on facial expression changes. Journal of Neural Engineering, 11(3), 1-12. http://dx.doi.org/10.1088/1741-2560/11/3/036004

Malach, R., Levy, I., \& Hasson, U. (2002). The topography of high-order human object areas. Trends in Cognitive Sciences, 6, (11), 176-184.

McCarthy, G., Puce, A., Gore, J.C., \& Allison, T. (1997). Face-specific processing in the human fusiform gyrus. Journal of Cognitive Neuroscience, 9, (5), 605-610. 
Münßinger, J.I., Halder, S., Kleih, S.C., Furdea, A., Raco, V., Hosle, A., \& Kubler, A. (2010). Brain painting: First evaluation of a new brain-computer interface application with ALSpatients and healthy volunteers. Frontiers in Neuroscience 4, (182), 1-11.

Picton, T. W. (1992). The P300 wave of the human event-related potential. Journal of Clinical Neurophysiology, 9, 456-79.

Pierce, J.R. (1980). An introduction to information theory. Dover, New York, pp. 145-165.

Rouder, J. N., Speckman, P. L., Sun, D., Morey, R. D., \& Iverson, G. (2009). Bayesian t-tests for accepting and rejecting the null hypothesis. Psychonomic Bulletin and Review, 16(2), 225-237. Retrieved from http://pcl.missouri.edu/sites/default/files/Rouder.bf_.pdf

Sellers, E.W., \& Donchin, E. (2006). A P300-based brain computer interface: Initial tests by ALS patients. Clinical Neurophysiology, 117, 538-548.

Sellers, E.W., Krusienski, D.J., McFarland, D.J., Vaughan, T.M., \& Wolpaw, J.R. (2006). P300 event-related potential brain-computer interface (BCI): The effects of matrix size and inter stimulus interval on performance. Biological Psychology, 73, 242-252.

Sajda, P., Müller, K., \& Shenoy, K.V. (2008, January). Brain-Computer Interfaces. IEEE Signal Processing Magazine (25), 1, 16-17.

Sutton S., Braren M., Zubin, J., \& John, E. R. (1965). Evoked potential correlates of stimulus uncertainty. Science, 150, 1187-8.

Takano, K., Komatsu, T., Hata, N., Nakajima, Y., \& Kansaku, K. (2009). Visual stimuli for the P300 brain-computer interface: A comparison of white/gray and green/blue flicker matrices. Clinical Neurophysiology, http://dx.doi.org/10.106/clinph.2009.06.002 
Tong, F., Nakayama, K., Vaughan, J.T., \& Kanwisher, N. (1998). Binocular rivalry and visual awareness in human extrastriate cortex. Neuron, 21, 753-759.

Touryan, J., Gibson, L., Horne, J. H., \& Weber, P. (2011) Real-time measurement of face recognition in rapid serial visual presentation. Front Psychology 2, (42). http://dx.doi.org/10.3389/fpsyg.2011.00042

Tucker, M. \& Ellis, R. (2004). Action priming by briefly presented objects. Acta Psychologica, 116, (2), 185-203. http://dx.doi.org/10.1016/j.actpsy.2004.01.004

Velliste, M., Perel, S., Spalding, M.C., Whitford, A.S., \& Schwartz, A.B. (2008). Cortical control of a prosthetic arm for self-feeding. Nature, 453, 1098-1101.

Vallabhaneni, A., Wang, T., \& He, B. (2005). Brain-computer interface. Neural Engineering, 85121. doi: 10.1007/0-306-48610-5_3

Zhang, Y., Zhao, Q., Jin, J., Wang, X., \& Cichocki, A. (2012). A novel BCI based on ERP components sensitive to configural processing of human faces. Journal of Neural Engineering, 2, (9), 1-13. 026018. http://dx.doi.org/10.1088/1741-2560/9/2/026018 


\section{VITA}

\section{MARISSA JONES}

Education:

Professional Experience: Graduate Assistant, East Tennessee State University, Johnson City, Tennessee, 2015-2017 